Engineering Physics and Mathematics Division

Mathematical Sciences Section

\title{
ARE BILINEAR QUADRILATERALS BETTER THAN LINEAR TRIANGLES?
}

\author{
E. F. D'Azevedo
}

Mathematical Sciences Section

Oak Ridge National Laboratory

P.O. Box 2008, Bldg. 6012

Oak Ridge, TN 37831-6367

Date Published: August 1993

Research supported by the Applied Mathematical Sciences subprogram of the Office of Energy Research, U.S. Department of Energy and in part by the Information Technology Research Centre, which is funded by the Province of Ontario.

Prepared by the

Oak Ridge National Laboratory

Oak Ridge, Tennessee 37831

managed by

Martin Marietta Energy Systems, Inc.

for the

U.S. DEPARTMENT OF ENERGY

under Contract No. DE-AC05-84OR21400
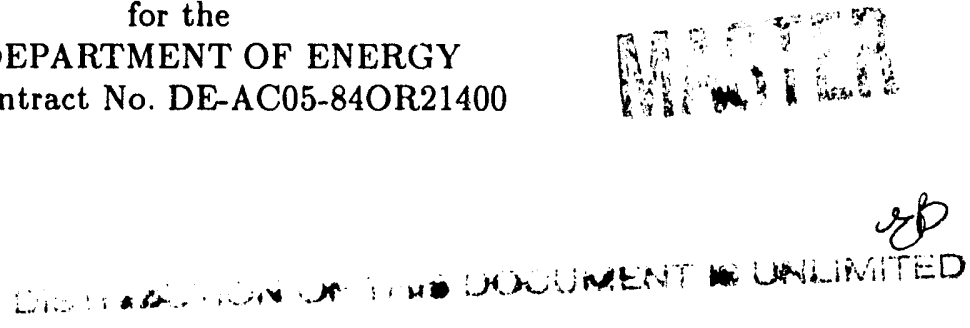


\section{Contents}

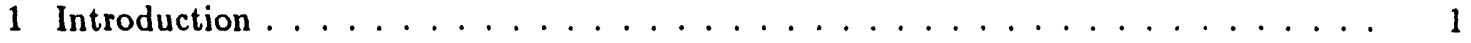

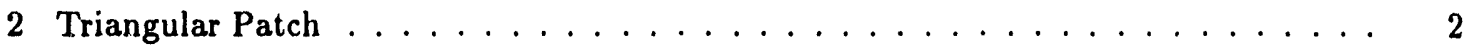

2.1 Quadratic Model .......................... 2

2.2 Optimal shape ........................... 3

2.3 Differential Geometry ....................... 4

3 Quadrilateral Patch ............................ 5

3.1 Restriction to Parallelogram . . . . . . . . . . . . . . . . 6

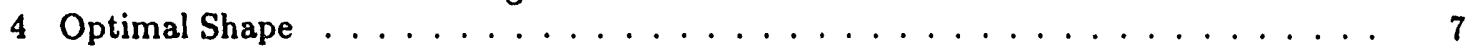

5 Comparison of quadrilaterals versus triangles . . . . . . . . . . . . . . 8

6 Extensions to three dimensions . . . . . . . . . . . . . . . . . . . 12

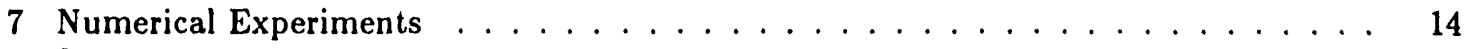

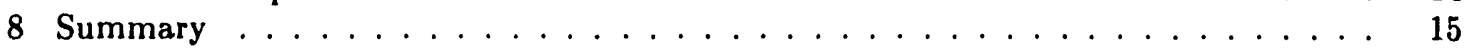

9 References . . . . . . . . . . . . . . . . . . . . . 23 


\section{List of Tables}

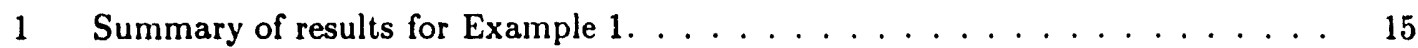

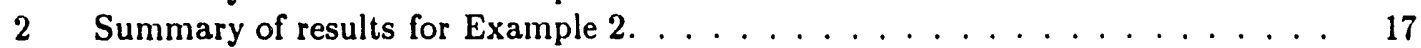

3 Summary of results for Example 3. . . . . . . . . . . . . . . . . . . . 17

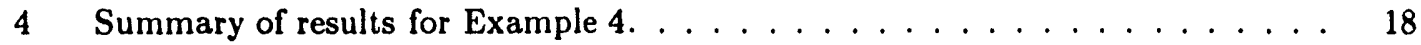

5 Convergence test on Example 3. . . . . . . . . . . . . . . 18 


\section{List of Figures}

Quadrilateral composed of two equilateral triangles. . . . . . . . . . . 9

Diagonal BD chosen to decompose quadrilateral. . . . . . . . . . . . . . . 9

Decomposition of a square. . . . . . . . . . . . . . . . 10

Triangulating a general parallelogram. . . . . . . . . . . . 11

Error profiles for Example 1. . . . . . . . . . . . . . . . . 15

Error profiles for Example 2. . . . . . . . . . . . . . . . . 16

Error profiles for Example 2. . . . . . . . . . . . . . . . . 16

Error profiles for Example $3 . \ldots \ldots \ldots \ldots 17$

Mesh I for Example 1. . . . . . . . . . . . . . . . . 18

10 Mesh II for Example 1. . . . . . . . . . . . . . . . . . . . . 19

11 Mesh I for Example 2. . . . . . . . . . . . . . . . . . . . 19

12 Mesh II for Example 2. . . . . . . . . . . . . . . . . . . . . . 20

13 Mesh I for Example 3. . . . . . . . . . . . . . . . . . . . . . 20

14 Mesh II for Example 3. . . . . . . . . . . . . . . . . . . . . 21

15 Mesh I for Example 4. ....................... 21

16 Mesh II for Example 4. . . . . . . . . . . . . . . . . . . . . . . 22 


\title{
ARE BILINEAR QUADRILATERALS BETTER
}

\section{THAN LINEAR TRIANGLES?}

\author{
E. F. D'Azevedo
}

\begin{abstract}
This paper compares the theoretical effectiveness of bilinear approximation over quadrilaterals with linear approximation over triangles. Anisotropic mesh transformation is used to generate asymptotically optimally efficient meshes for piecewise linear interpolation over triangles and bilinear interpolation over quadrilaterals. The theory and numerical results suggest triangles may have a slight advantage over quadrilaterals for interpolating convex data function but bilinear approximation may offer a higher order approximation for saddle-shaped functions on a well-designed mesh.
\end{abstract}

$-\mathrm{ix}-$ 


\section{Introduction}

This paper compares the theoretical effectiveness of bilinear approximation over quadrilaterals with linear approximation over triangles. The novelty is in the use of anisotropic mesh transformation to generate asymptotically optimally efficient meshes in the comparison. This work is a basic study on optimal meshes with the intention of gaining insight into the more complex meshing problems in ñnite element analysis. The theory and numerical results suggest triangles may have a slight advantage over quadrilaterals for interpolating convex data functions but bilinear approximation may offer a higher order approximation for saddle-shaped functions on a well-designed mesh.

We consider the problem of interpolating a given smooth data function with continuous piecewise linear triangles or bilinear quadrilaterals over a domain to satisfy a given error tolerance. A mesh that achieves this error tolerance with the fewest elements is defined to be optimally efficient. Intuitively, one would expect smaller and denser elements in regions where the function has sharp peaks or large variations. Since each convex quadrilateral can be split across either one of the diagonals into two triangles, one can imagine embedding a refined triangular mesh within the quadrilateral mesh. A question arises as to whether the bilinear approximation over quadrilaterals or linear approximation over triangles is more effective.

To make a fair comparison, we need to compare bilinear approximation over an "optimal" quadrilateral mesh versus linear approximation over an "optimal" triangular mesh. Indeed, $\S 5$ contains an example where the way quadrilaterals are decomposed into triangles may yield different interpolation accuracies. Provably optimal triangular meshes $[2,4]$ have been produced by anisotropic mesh transformation.

Anisotropic mesh transformation is emerging as an effective technique for unstructured grid generation where the vertex distribution is highly non-uniform. The central idea is to control the element shapes and sizes by specifying a symmetric metric tensor that measures the approximation error. The metric tensor determines the corresponding anisotropic transformation. The anisotropic mesh is then the image of a uniform mesh of optimal shape elements under the anisotropic transformation. Simpson [8] gives a survey on anisotropic meshes.

Nadler [6], D'Azevedo and Simpson [3,4], and D'Azevedo [2] have studied local anisotropic transformation for the generating of optimally efficient triangular meshes. Peraire et al. [7] applied anisotropic transformation in mesh generation for dynamic remeshing in solving compressible flow problems. In these works, piecewise linear approximation of a quadratic function is used as the model for local analysis. In this paper we extend a similar analysis to bilinear approximation on quadrilateral patches.

An outline of the paper follows. In $§ 2$, we review the key ideas in [2] for generating optimally efficient triangular meshes. In $§ 3$, we consider error properties of bilinear interpolation. We 
consider the optimal geometry for quadrilateral patches in $\S 4$. We compare the effectiveness of quadrilaterals versus triangular meshes using the local quadratic model in $\$ 5$. Section 6 contains extensions to hexahedral bricks in three dimensions. Numerical experiements and the results are described in $\S 7$. Finally $\S 8$ gives a brief summary.

\section{Triangular Patch}

In this section, we review the basic techniques in determining optimal triangle geometry used in [2]. We show a linear transformation of a regular mesh of optimal-shape triangles yields an optimally efficient mesh for interpolating a quadratic function.

\subsection{Quadratic Model}

Given the data values at the three vertices of a triangle, the piecewise linear inter jlant

$$
p_{\ell}(x, y)=p_{0}+p_{1} x+p_{2} y, \quad p_{\ell}\left(x_{i}, y_{i}\right)=f_{i}
$$

can be determined by solving the $3 \times 3$ system of linear equations,

$$
\left[\begin{array}{lll}
1 & x_{1} & y_{1} \\
1 & x_{2} & y_{2} \\
1 & x_{3} & y_{3}
\end{array}\right]\left[\begin{array}{l}
p_{0} \\
p_{1} \\
p_{2}
\end{array}\right]=\left[\begin{array}{l}
f_{1} \\
f_{2} \\
f_{3}
\end{array}\right]
$$

Note the determinant of the above matrix is two times the area of the triangle, hence we have a nonsingular system for a nondegenerate triangle.

We shall consider a local analysis where we assume the data function $f(x, y)$ is well approximated by its quadratic Taylor expansion,

$$
f(x+d x, y+d y) \approx f(x, y)+\nabla f(x, y)[d x, d y]+\frac{1}{2}[d x, d y] H(x, y)[d x, d y]^{t} .
$$

Let the error formula be $E_{T}(x, y)=p_{\ell}(x, y)-f(x, y)$. By our assumption, $E_{\Upsilon^{\prime}}(x, y)$ is a quadratic function and level curves for $E_{T}(x, y)=c$ form a family of conics with a common center at $\left(x_{c}, y_{c}\right)$. They form a family of ellipses if $\operatorname{det}(H)>0$, and hyperbolas if $\operatorname{det}(H)<0$. Note by the interpolation condition, the curve $E_{T}(x, y)=0$ passes through all vertices of the triangle. If $\operatorname{det}(H)>0$ (conic is ellipse) then $E_{T}(x, y)$ attains the local maximum at the center $\left(x_{c}, y_{c}\right)$; otherwise, $\operatorname{det}(H)<0$ (conic is hyperbola) the maximum error is attained at the midpoint of an edge. The error at a displacement from the center is given by

$$
E_{T}\left(x_{c}+d x, y_{c}+d y\right)=\mathcal{E}_{T}-\frac{1}{2}[d x, d y] H[d x, d y]^{t}, \quad \mathcal{E}_{T}=E_{T}\left(x_{c}, y_{c}\right)
$$


The key insight in [2] is in interpreting the Hessian matrix $H$ in (2) as a symmetric metric tensor. Let the symmetric Hessian matrix be diagonalizable as

$$
\begin{aligned}
H & =Q^{t}\left[\begin{array}{cc}
\lambda_{1} & 0 \\
0 & \lambda_{2}
\end{array}\right] Q=S^{t}\left[\begin{array}{ll}
1 & 0 \\
0 & \epsilon
\end{array}\right] S, \quad \text { where } \epsilon=\operatorname{sign}(\operatorname{det}(H)) \\
S & =\left[\begin{array}{cc}
\sqrt{\left|\lambda_{1}\right|} & 0 \\
0 & \sqrt{\left|\lambda_{2}\right|}
\end{array}\right] Q
\end{aligned}
$$

Under this transformation $S$, the expression $[d x, d y] H[d x, d y]^{t}$ reduces to $(d \tilde{x})^{2}+\epsilon(d \tilde{y})^{2}$, where $[\tilde{x}, \tilde{y}]^{t}=S[x, y]^{t}$. The error function can be rewritten as

$$
\begin{aligned}
E_{T}\left(x_{c}+d x, y_{c}+d y\right) & =\mathcal{E}_{T}-\frac{1}{2}[d x, d y] H[d x, d y]^{t} \\
& =\mathcal{E}_{T}-\frac{1}{2}\left((d \tilde{x})^{2}+\epsilon(d \tilde{y})^{2}\right) \\
& =\tilde{E}_{T}\left(\tilde{x}_{c}+d \tilde{x}, \tilde{y}_{c}+d \tilde{y}\right)
\end{aligned}
$$

where $\tilde{E}_{T}(\tilde{x}, \tilde{y})$ dcnotes the corresponding error function under transformation $S$ in $(\tilde{x}, \tilde{y})$-space. The error expression $\tilde{E}_{T}(\tilde{x}, \tilde{y})$ has no preferred direction hence we shall call the $(\tilde{x}, \tilde{y})$-space the "isotropic" space.

\subsection{Optimal shape}

In the following, we shall determined the best triangle shape that minimizes the interpolation error. We can determine the "efficiency" of the elements by computing their ratio of Error to Area. A small ratio indicates a more efficient element, i.e. one can achieve a lower error tolerance and tile the domain with about the same number of elements.

We consider first the case $f(x, y)$ is convex $(\operatorname{det}(H)>0, \epsilon=1)$ and level curves or contours of $\tilde{E}_{T}(\tilde{x}, \tilde{y})$ are concentric circles given by

$$
\tilde{E}_{T}\left(\tilde{x}_{c}+d \tilde{x}, \tilde{y}_{c}+d \tilde{y}\right)=\mathcal{E}_{T}-\frac{1}{2}\left((d \tilde{x})^{2}+(d \tilde{y})^{2}\right)
$$

Let $\tilde{T}$ be the transformed of triangle $T$ over the isotropic space, with vertices at $\left(\tilde{x}_{1}, \tilde{y}_{1}\right),\left(\tilde{x}_{2}, \tilde{y}_{2}\right)$ and $\left(\tilde{x}_{3}, \tilde{y}_{3}\right)$. The circumcircle of $\tilde{T}$ corresponds to the level curve of value zero. Hence the radius of this circumcircle is $\operatorname{sqrt}\left(2\left|\mathcal{E}_{T}\right|\right)$ and relates directly to the maximum error attainable (at the center). We can easily see that an equilateral triangle covers the most area for a fixed circumcircle; therefore an equilateral triangle for $\tilde{T}$ is of optimal-shape.

Consider next the case where $f(x, y)$ is not convex but has a saddle-shaped $\operatorname{graph}(\operatorname{det}(H)<$ 
$0, \epsilon=-1)$, then we have

$$
\begin{aligned}
\tilde{E}_{T}(\tilde{x}, \tilde{y}) & =\tilde{E}_{T}\left(\tilde{x}_{c}+d \tilde{x}, \tilde{y}_{c}+d \tilde{y}\right) \\
& =\mathcal{E}_{T}-\frac{1}{2}\left((d \tilde{x})^{2}-(d \tilde{y})^{2}\right) \\
& =\mathcal{E}_{T}-\frac{1}{2}\left(\left(\tilde{x}-\tilde{x}_{c}\right)^{2}-\left(\tilde{y}-\tilde{y}_{c}\right)^{2}\right)
\end{aligned}
$$

We note that the error function $\tilde{E}_{T}(\tilde{x}, \tilde{y})$ is a harmonic function and attains its extrema on the boundary of $\tilde{T}$. By calculus, we can show that the local extrema along edge $\left(\tilde{x}_{i}, \tilde{y}_{i}\right),\left(\tilde{x}_{j}, \tilde{y}_{j}\right)$ is attained at the midpoint with value

$$
\tilde{E}\left(\frac{\tilde{x}_{i}+\tilde{x}_{j}}{2}, \frac{\tilde{y}_{i}+\tilde{y}_{j}}{2}\right)=\frac{1}{8}\left|\left(\tilde{x}_{i}-\tilde{x}_{j}\right)^{2}-\left(\tilde{y}_{i}-\tilde{y}_{j}\right)^{2}\right|
$$

The details for finding the optimal-shape triangle in this case are found in [4]. The optimalshape triangle geometry that minimizes the efficiency ratio (Error/Area) is not unique. The same maximum error is attained at the mid-point of each edge.

From the above two results on optimal-shape triangles, we see that a regular mesh of optimalshape triangles over the isotropic $(\tilde{x}, \tilde{y})$-space corresponds to an optimally efficient mesh over the original $(x, y)$-space. Every triangle attains the same maximum error; moreover, these triangles cover the most area for the error attained and so are optimally efficient. Since the linear transformation $S$ is basically a rotation followed by a rescaling of coordinate axes, we find the areas of triangles are scaled accordingly. Hence the inverse transformation $S^{-1}$, maps this regular mesh to produce an optimally efficient mesh in the original $(x, y)$-space.

\subsection{Differential Geometry}

The constant Hessian Matrix $H$ in (1) determines the coordinate transformation $S$ that maps $[\tilde{x}, \tilde{y}]^{t}=S[x, y]^{t}$ so that

$$
[d x, d y] H[d x, d y]^{t}=\left(d \tilde{x}^{2}+\epsilon d \tilde{y}^{2}\right)
$$

More generally, we may view $H$ as a metric tensor for measuring the interpolation orror $[d x, d y] H[d x, d y]^{t}$. Thus we need to determine $(\tilde{x}(x, y), \tilde{y}(x, y))$, a continuous transformation that satisfies $[d x, d y] H[d x, d y]^{t}=d \tilde{x}^{2}+\epsilon d \tilde{y}^{2}$. The conditions for finding the anisotropic coordinate transformation $(\tilde{x}(x, y), \tilde{y}(x, y))$ are given by a classical result in differential geometry for characterizing a "flat" space [9]: that the Riemann-Christoffel tensor formed from the metric tensor $H$ is identically zero. In our case, a sufficient condition is for $H=\left\{h_{i j}\right\}$ to satisfy

$$
K_{1} h_{11}+K_{2} h_{12}+K_{3} h_{22}=0
$$


for some constants $K_{1}, K_{2}, K_{3}$. The coordinate transformation $(\tilde{x}(x, y), \tilde{y}(x, y))$ may be found by solving an initial value ordinary differential equation. Again, the inverse transformation $(x(\tilde{x}, \tilde{y}), y(\tilde{x}, \tilde{y}))$ maps a regular mesh of optimal shaped triangles to yield an optimally efficient mesh.

\section{Quadrilateral Patch}

In this section, we derive the error term for bilinear approximation of a quadratic data function. We first consider a rectangle $\left(x_{i}, y_{i}\right), i=1 \ldots 4$ with sides aligned along the coordinate axes. To evaluate the bilinear interpolant $p_{\ell}(x, y)$, we compute

$$
\begin{aligned}
p & =\frac{x-x_{1}}{x_{4}-x_{1}}=\frac{x-x_{2}}{x_{3}-x_{2}}, \quad q=\frac{y-y_{1}}{y_{4}-y_{1}}=\frac{y-y_{2}}{y_{3}-y_{2}} \\
p_{\ell}(x, y) & =(1-p)\left[q f_{4}+(1-q) f_{1}\right]+p\left[q f_{3}+(1-q) f_{2}\right], \quad \text { where } f_{i}=f\left(x_{i}, y_{i}\right)
\end{aligned}
$$

Note the existence of $x y$ terms from $p q$ and $p_{\ell}(x, y)$ is linear on each side. The linear function is determined by the function values at the corresponding vertices.

For a general quadrilateral, it is impossible to find a polynomial in $x$ and $y$ which reduces to an arbitrary linear form along the four sides [5]. Hence simply solving for the undetermined coefficients in $p_{\ell}(x, y)=p_{0}+p_{1} x+p_{2} y+p_{12} x y$ as done for triangles by imposing the interpolation condition

$$
\left[\begin{array}{llll}
1 & x_{1} & y_{1} & x_{1} y_{1} \\
1 & x_{2} & y_{2} & x_{2} y_{2} \\
1 & x_{3} & y_{3} & x_{3} y_{3} \\
1 & x_{4} & y_{4} & x_{4} y_{4}
\end{array}\right]\left[\begin{array}{c}
p_{0} \\
p_{1} \\
p_{2} \\
p_{12}
\end{array}\right]=\left[\begin{array}{c}
f_{1} \\
f_{2} \\
f_{3} \\
f_{4}
\end{array}\right]
$$

might fail with a singular matrix.

To overcome this limitation, we shall use the isoparametric formulation (commonly used in finite element analysis) by considering basis functions over the normalized $(p, q)$-space over the unit square, $0 \leq p, q \leq 1$. Basis functions are

$$
\begin{array}{ll}
\phi_{1}(p, q)=(1-p)(1-q), & \phi_{2}(p, q)=p(1-q) \\
\phi_{3}(p, q)=p q, & \phi_{4}(p, q)=(1-p) q
\end{array}
$$

Note that $\phi_{1}(0,0)=1, \phi_{1}(p, q)=0$ at the other corners and the basis functions sum to one, $\sum_{i=1}^{i=4} \phi_{i}(p, q)=1$. 
Mapping from $(p, q)$ to the original $(x, y)$-space is by

$$
\begin{aligned}
& x(p, q)=x_{1} \phi_{1}(p, q)+x_{2} \phi_{2}(p, q)+x_{3} \phi_{3}(p, q)+x_{4} \phi_{4}(p, q) \\
& y(p, q)=y_{1} \phi_{1}(p, q)+y_{2} \phi_{2}(p, q)+y_{3} \phi_{3}(p, q)+y_{4} \phi_{4}(p, q)
\end{aligned}
$$

that maps vertex $(0,0)$ to $\left(x_{1}, y_{1}\right)$, vertex $(1,0)$ to $\left(x_{2}, y_{2}\right),(1,1)$ to $\left(x_{3}, y_{3}\right)$ and $(0,1)$ to $\left(x_{4}, y_{4}\right)$. The bilinear interpolant (over $(p, q)$-space) is given by

$$
p_{\ell}(x(p, q), y(p, q))=f_{1} \phi_{1}(p, q)+f_{2} \phi_{2}(p, q)+f_{3} \phi_{3}(p, q)+f_{4} \phi_{4}(p, q), \quad f_{i}=f\left(x_{i}, y_{i}\right)
$$

\subsection{Restriction to Parallelogram}

The isoparametric formulation maps from the canonical $(p, q)$-space to the original $(x, y)$-space. For a general quadrilateral patch, it may be difficult to generate the inverse transformation $(p(x, y), q(x, y))$ from the original $(x, y)$ space to the canonical $(p, q)$ space. From (7) and $(8)$, :ve have

$$
x(p, q)=\left(x_{1}-x_{2}+x_{3}-x_{4}\right) p q+\left(x_{2}-x_{1}\right) p+\left(x_{4}-x_{1}\right) q+x_{1},
$$

and a similar expression for $y(p, q)$. The isoparametric map is an affine transformation if and only if $\left(x_{1}-x_{2}+x_{3}-x_{4}\right)=0$ and $\left(y_{1}-y_{2}+y_{3}-y_{4}\right)=0$, i.e. the quadrilateral patch has parallel sides. Let the parallel sides be

$$
\left[\begin{array}{l}
u_{x} \\
u_{y}
\end{array}\right]=\left[\begin{array}{l}
x_{2}-x_{1} \\
y_{2}-y_{1}
\end{array}\right]=\left[\begin{array}{l}
x_{3}-x_{4} \\
y_{3}-y_{4}
\end{array}\right], \quad\left[\begin{array}{l}
v_{x} \\
v_{y}
\end{array}\right]=\left[\begin{array}{c}
x_{4}-x_{1} \\
y_{4}-y_{1}
\end{array}\right]=\left[\begin{array}{l}
x_{3}-x_{2} \\
y_{3}-y_{2}
\end{array}\right],
$$

then (8) simplifies to the affine trar-formation

$$
\left[\begin{array}{l}
x(p, q) \\
y(p, q)
\end{array}\right]=\left[\begin{array}{ll}
u_{x} & v_{x} \\
u_{y} & v_{y}
\end{array}\right]\left[\begin{array}{l}
p \\
q
\end{array}\right]+\left[\begin{array}{l}
x_{1} \\
y_{1}
\end{array}\right]
$$

The affine transformation is well defined since the area of the quadrilateral patch is

$$
\mathcal{A}=\left(u_{x} v_{y}-u_{y} v_{x}\right)=\operatorname{det}\left(\left[\begin{array}{ll}
u_{x} & v_{x} \\
u_{y} & v_{y}
\end{array}\right]\right) \neq 0 \text {. }
$$

Since we intend to apply coordinate transformation to a regular quadrilateral mesh in the generation of asymptotically optimal meshes, the consideration of only parallelograms is not a severe restriction. 


\section{Optimal Shape}

In the following, we shall determine the best parallelogram shape that minimizes the interpolation error. The error function for quadratic interpolation is

$$
\begin{aligned}
E_{Q}(p, q) & =p_{\ell}(x(p, q), y(p, q))-f(x(p, q), y(p, q)) \\
& =\mathcal{E}_{Q}-\frac{1}{2}\left(\mu_{1} d p^{2}+\mu_{2} d q^{2}\right)
\end{aligned}
$$

where $\left[p_{c}, q_{c}\right]=[1 / 2,1 / 2]$,

$$
\begin{aligned}
\mathcal{E}_{Q} & =E_{Q}\left(p_{c}, q_{c}\right)=\frac{1}{8}\left(\mu_{1}+\mu_{2}\right) \\
0 & =\frac{\partial}{\partial p} E_{Q}\left(p_{c}, q_{c}\right)=\frac{\partial}{\partial q} E_{Q}\left(p_{c}, q_{c}\right) \\
\mu_{1} & =\left[u_{x}, u_{y}\right] H\left[u_{x}, u_{y}\right]^{t}, \quad \mu_{2}=\left[v_{x}, v_{y}\right] H\left[v_{x}, v_{y}\right]^{t}, .
\end{aligned}
$$

For a convex function $(\operatorname{det}(H)>0), \mu_{1}$ and $\mu_{2}$ are positive, hence the maximum error is attained at the center $\left[p_{c}, q_{c}\right]$.

Under the aniso'ropic transformation, we have

$$
\begin{array}{ll}
{\left[\tilde{u}_{x}, \tilde{u}_{y}\right]^{t}=S\left[u_{x}, u_{y}\right]^{t},} & {\left[\tilde{v}_{x}, \tilde{v}_{y}\right]^{t}=S\left[v_{x}, v_{y}\right]^{t}} \\
\mu_{1}=\tilde{u}_{x}^{2}+\tilde{u}_{y}^{2}=L_{1}^{2}, & \mu_{2}=\tilde{v}_{x}^{2}+\tilde{v}_{y}^{2}=L_{2}^{2}
\end{array}
$$

The area of the quadrilateral in isotropic space is

$$
\tilde{\mathcal{A}}=\tilde{u}_{x} \tilde{v}_{y}-\tilde{u}_{y} \tilde{v}_{x}=L_{1} L_{2} \sin (\theta)
$$

where $\theta$ is the angle at corner $\left[\tilde{x}_{1}, \tilde{y}_{1}\right]$. Since the anisotropic transformation $S$ is a rotation followed by a rescaling of coordinate axis (3),

$$
S=\left[\begin{array}{cc}
\sqrt{\left|\lambda_{1}\right|} & 0 \\
0 & \sqrt{\left|\lambda_{2}\right|}
\end{array}\right] Q
$$

the area of quadrilateral over isotropic space is $\operatorname{sqrt}\left(\left|\lambda_{1} \lambda_{2}\right|\right)=\operatorname{sqrt}(|\operatorname{det}(H)|)$ times the area of the quadrilateral over the original $(x, y)$ space,

$$
\tilde{\mathcal{A}}=\mathcal{A} \sqrt{\left|\lambda_{1} \lambda_{2}\right|}=\mathcal{A} \sqrt{|\operatorname{det}(H)|}
$$

Note that $\operatorname{det}(H)$ is intrinsic to the data function $f(x, y)$.

A square (in any orientation) with $L_{1}=L_{2}$ and $\sin (\theta)=1$ minimizes the efficiency ratio 
(Error/Area),

$$
\frac{\mathcal{E}_{Q}}{\tilde{A}}=\frac{1}{8} \frac{\mu_{1}+\mu_{2}}{L_{1} L_{2} \sin (\theta)}=\frac{1}{8} \frac{L_{1}^{2}+L_{2}^{2}}{L_{1} L_{2} \sin (\theta)} \leq \frac{1}{4},
$$

and hence is of optimal shape.

If $f(x, y)$ is saddle-shaped $(\operatorname{det}(H)<0)$ the error expression is still

$$
E_{Q}(p, q)=\frac{1}{8}\left(\mu_{1}+\mu_{2}\right)-\frac{1}{2}\left(\mu_{1} d p^{2}+\mu_{2} d q^{2}\right) .
$$

Under the anisotropic transformation $S$,

$$
\mu_{1}=\tilde{u}_{x}^{2}-\tilde{u}_{y}^{2}, \quad \mu_{2}=\tilde{v}_{x}^{2}-\tilde{v}_{y}^{2} .
$$

Both $\mu_{1}$ and $\mu_{2}$ vanish for

$$
\left[\tilde{u}_{x}, \tilde{u}_{y}\right]=[L, L], \quad\left[\tilde{v}_{x}, \tilde{v}_{y}\right]=[-L, L]
$$

which correspond to a square rotated by $\pi / 4$. The above indicates an "exact fit" $\left(E_{Q}(p, q)=0\right)$ if $\mu_{1}=\mu_{2}=0$. This suggests bilinear approximation is a better interpolant than simple linear interpolation and the simple quadratic model is inadequate to fully capture the error properties in this case.

To summarize, a square over the isotropic space in any orientation is optimal for the elliptic case, and a square rotated by $\pi / 4$ is optimal for the hyperbolic case.

\section{Comparison of quadrilaterals versus triangles}

In this section, we apply the geometric interpretation of the maximum interpolation error over the transformed isotropic space. We recall that over the isotropic space and for a convex data function $(\operatorname{det}(H)>0)$, the maximum error $\left(\mathcal{E}_{T}\right)$ for a triangle is attained at the center of its circum-circle with $\mathcal{E}_{T}=R^{2} / 2$, where $R$ is radius of the circum-circle. The maximum error $\left(\mathcal{E}_{Q}\right)$ for a parallelogram is attained at its centroid with $\mathcal{E}_{Q}=\left(L_{1}^{2}+L_{2}^{2}\right) / 8$ where $L_{1}, L_{2}$ are lengths of the sides.

Case 1. Consider the quadrilateral produced by merging two equilateral triangles of length $L$ (see Fig. 1). Radius of circumcircle of $\triangle A B C$ is $R=L / \sqrt{3}$, thus

$$
\mathcal{E}_{T}=R^{2} / 2=L^{2} / 6, \quad \mathcal{E}_{Q}\left(L^{2}+L^{2}\right) / 8=L^{2} / 4
$$

Thus $\mathcal{E}_{Q}>\mathcal{E}_{T}$, so triangles are more accurate. 


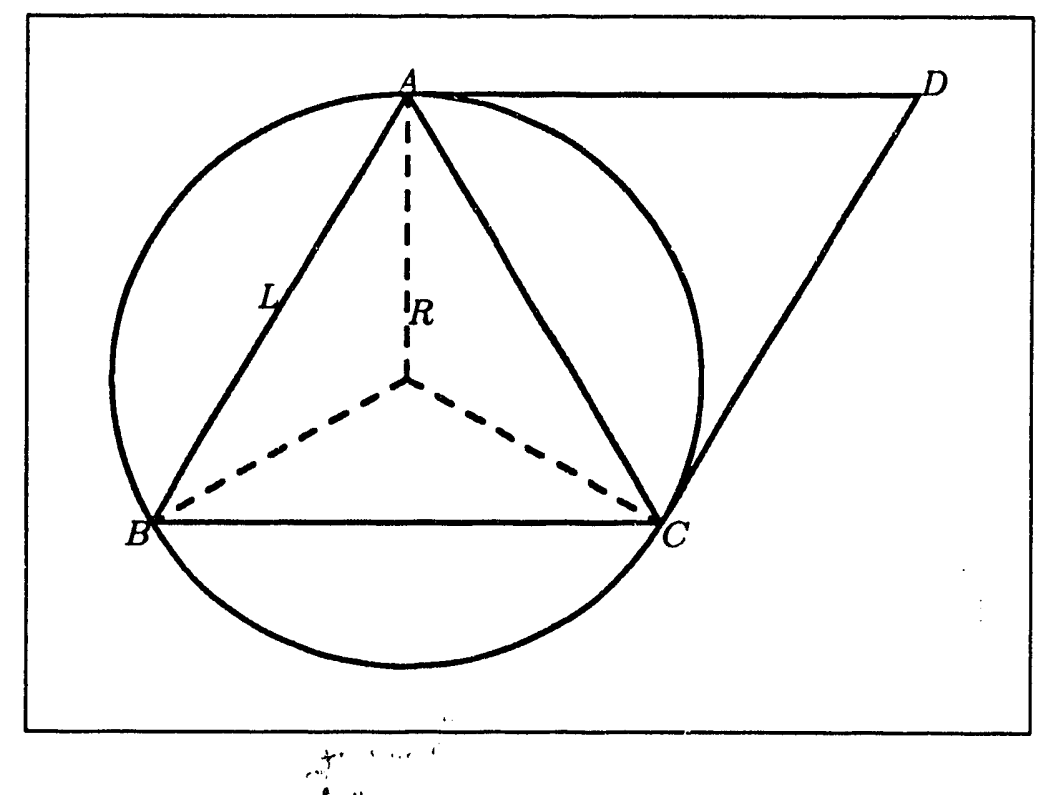

Figure 1: Quadrilateral composed of two equilateral triangles.

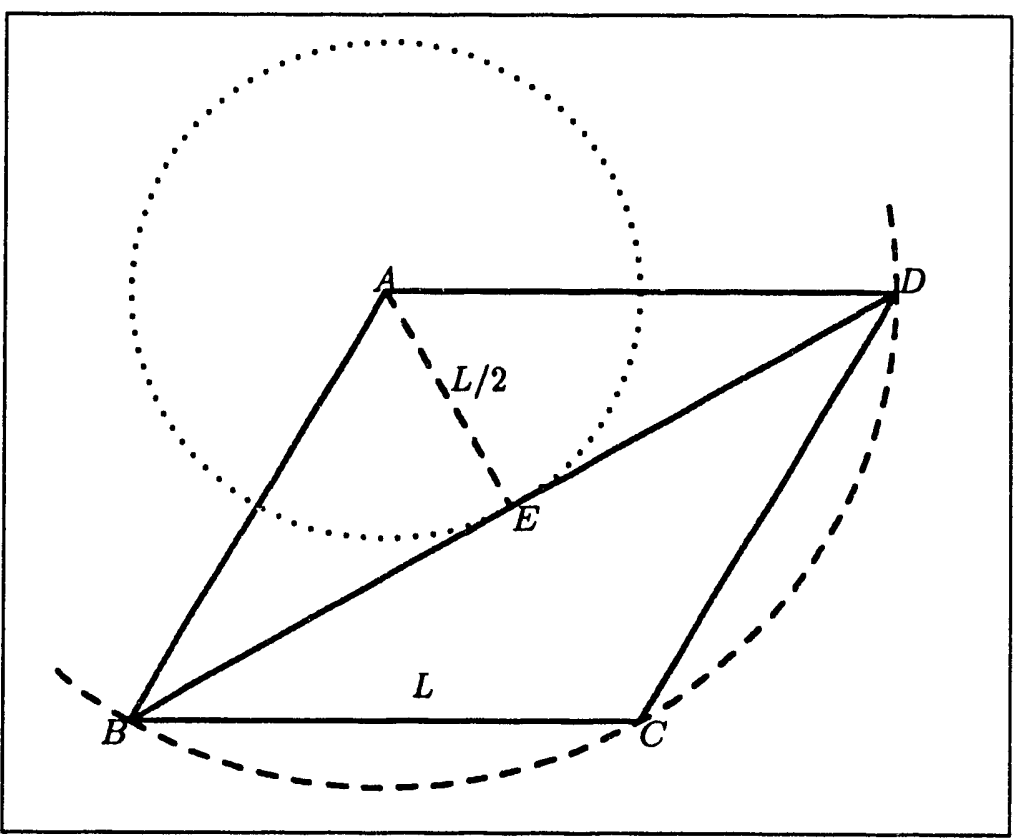

Figure 2: Diagonal BD chosen to decompose quadrilateral. 
An earlier work [3] has shown the optimal triangulation incidence is given by the Delaunay triangulation over the isotropic space. If we consider the same quadrilateral with the "wrong" decomposition with diagonal $B D$ (see Fig. 2), the maximum error $\triangle B C D$ is attained at vertex $E$ with value $\hat{\mathcal{E}}_{T}$. Note vertex $A$ is the center of circumcircle for $\triangle B C D$ with radius $L$. The error attained at vertex $E$ is

$$
\begin{aligned}
\hat{\mathcal{E}}_{T} & =\mathcal{E}_{T}-\frac{1}{2}\left(d \tilde{x}^{2}+d \tilde{x}^{2}\right) \\
& =\left(L^{2} / 2\right)-\frac{1}{2}\left((L / 2)^{2}\right)=3 L^{2} / 8 .
\end{aligned}
$$

Thus $\hat{\mathcal{E}}_{T}=3 L^{2} / 8>L^{2} / 4=\mathcal{E}_{Q}$, and the quadrilateral is more accurate.

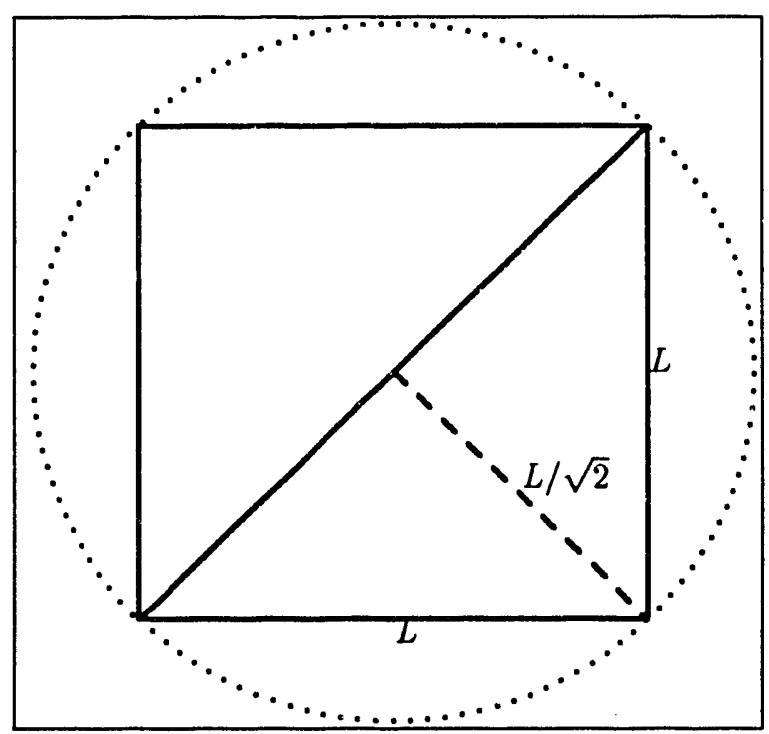

Figure 3: Decomposition of a square.

Case 2. Consider the triangulation produced by decomposing the optimal square configuration for quadrilaterals (see Fig. 3). Radius of circumcircle is $R=L / \sqrt{2}$,

$$
\mathcal{E}_{Q}=\left(L^{2}+L^{2}\right) / 8=L^{2} / 4, \quad \mathcal{E}_{T}=R^{2} / 2=L^{2} / 4
$$

Thus $\mathcal{E}_{Q}=\mathcal{E}_{T}$.

In fact we can show a stronger result that the refined triangulation will always produce better accuracy for approximating a convex function. Consider the parallelogram in Fig. 4 with $L_{1}=|B C|, L_{2}=|A B|, \angle A B C=\theta_{1}+\theta_{2} \leq \pi / 2$ and vertex $O$ is center of circumcircle for 


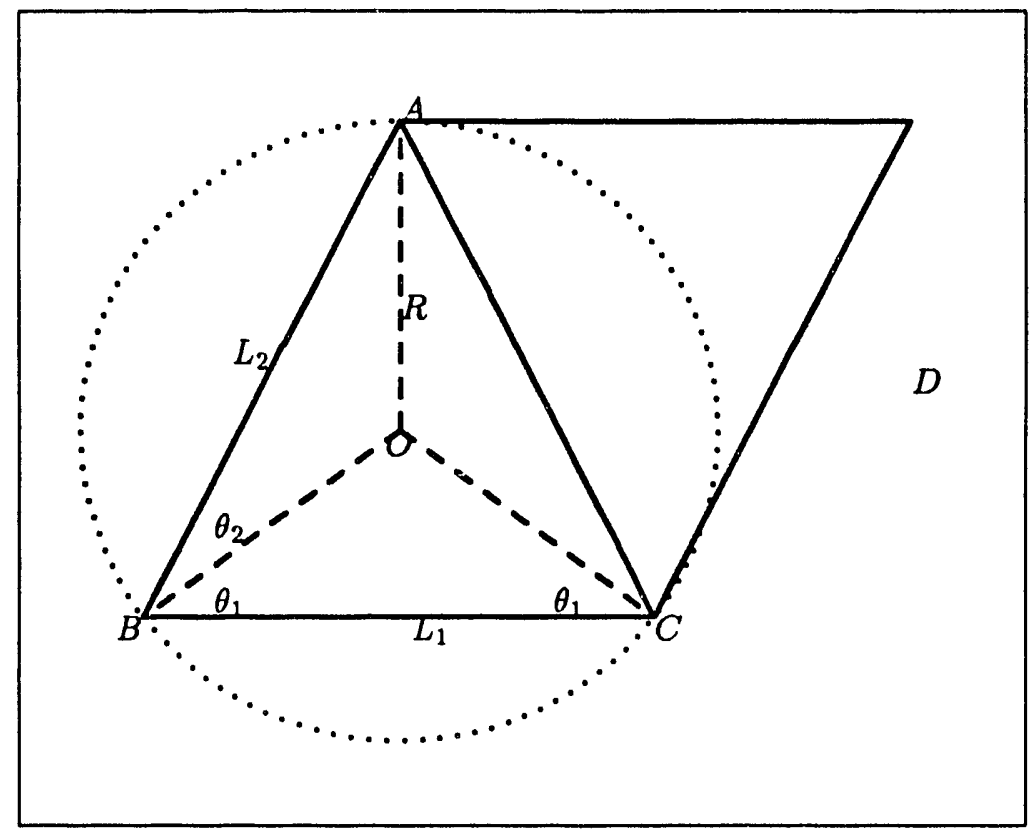

Figure 4: Triangulating a general parallelogram.

$\triangle A B C$ with radius $R$. By the sine rule over $\triangle O B C$ and $\triangle O A B$,

$$
\begin{gathered}
\frac{R}{\sin \left(\theta_{1}\right)}=\frac{L_{1}}{\sin \left(\pi-2 \theta_{1}\right)}, \quad \frac{R}{\sin \left(\theta_{2}\right)}=\frac{L_{2}}{\sin \left(\pi-2 \theta_{2}\right)} \\
2 R \cos \left(\theta_{1}\right)=L_{1}, \quad 2 R \cos \left(\theta_{2}\right)=L_{2}
\end{gathered}
$$

The error for this quadrilateral is

$$
\begin{aligned}
\mathcal{E}_{Q} & =\left(L_{1}^{2}+L_{2}^{2}\right) / 8 \\
& =\frac{R^{2}}{2}\left(\cos ^{2}\left(\theta_{1}\right)+\cos ^{2}\left(\theta_{2}\right)\right) \quad \text { by }(15) \\
& =\mathcal{E}_{T}\left(\cos ^{2}\left(\theta_{1}\right)+\cos ^{2}\left(\theta_{2}\right)\right) .
\end{aligned}
$$

Now $0<\theta_{1}+\theta_{2} \leq \pi / 2$, and $1 \leq\left(\cos ^{2}\left(\theta_{1}\right)+\cos ^{2}\left(\theta_{2}\right)\right) \leq 2$, so that $\mathcal{E}_{T} \leq \mathcal{E}_{Q} \leq 2 \mathcal{E}_{T}$.

From the above, (the area of equilateral triangle $\mathcal{A}_{T}$ is $\sqrt{3} L^{2} / 4$ ), we obtain an efficiency ratio of

$$
\frac{\mathcal{E}_{T}}{\mathcal{A}_{T}}=\frac{L^{2} / 6}{\sqrt{3} L^{2} / 4}=2 \sqrt{3} / 9 \approx 0.385
$$

Area of optimal square configuration is $L^{2}$, thus the ratio is $1 / 4=0.25$. Hence for an element 
by element comparison, the quadrilateral is more efficient. In other words, if we were to approximate a function with either $N$ quadrilaterals or $N$ triangles, quadrilaterals are preferred.

On the other hand, triangles may have advantages over quadrilaterals for finite element computations. Matrix assembly and the solution of the sparse linear equations are commonly the most intensive calculations. If we decompose a quadrilateral mesh into triangles as done above, no extra nodes are introduced. There will be twice as many triangular elements but the resulting assembled matrix has the identical sparsity pattern and the same number of unknowns. Matrix assembly with a general convex quadrilateral usually requires multiple costly evaluations of the Jacobian distortion in numerical quadrature over the isoparametric space, whereas only one preassembled element table is repeatedly reused for all triangles. Thus matrix assembly with triangles may be competitive with assembly with general quadrilaterals. In other words, if computation with $N$ quadrilaterals is as costly as using $2 N$ triangles, then triangles are preferred. The actual computation costs may depend on the implementation of the finite element code.

Case 3. Consider the approximation of a saddle-shaped function by a square (unrotated) over the isotropic space. The error formula gives

$$
\begin{aligned}
E_{Q}\left(p_{c}+d p, q_{c}+d q\right) & =\frac{1}{8}\left(\mu_{1}+\mu_{2}\right)-\frac{1}{2}\left(\mu_{1} d p^{2}+\mu_{2} d q^{2}\right), \quad\left(p_{c}, q_{c}\right)=\left(\frac{1}{2}, \frac{1}{2}\right) \\
& =-\frac{1}{2}\left(d p^{2} L^{2}-d q^{2} L^{2}\right), \quad \text { where } \mu_{1}=L^{2}=-\mu_{2},
\end{aligned}
$$

The maximum error is attained at the mid-point of each edge. Let $(d p, d q)=(1 / 2,0)$, then $\mathcal{E}_{Q}=L^{2} / 8$. This gives an efficiency ratio of $1 / 8=0.125$. One optimal triangle shape for saddle-shaped function is the triangle with vertices at $(0,0),(L, 0),(1 / 2 L, \sqrt{5} / 2 L)$ over the isotropic space [2], which has area $\sqrt{5} L^{2} / 4$. The maximum error is $L^{2} / 8$ and attained at the mid-point of each edge. This gives an efficiency ratio of $1 /(2 \sqrt{5}) \approx 0.224$. Thus a mesh with $N$ quadrilaterals should yield roughly the same accuracy as $2 N$ triangles. This is verified in the nunerical experiments.

\section{Extensions to three dimensions}

The previous results for quadrilaterals easily extend to trilinear interpolation over hexahedral bricks in three dimensions. Again we consider the isoparametric formulation,

$$
x(p, q, r)=\sum_{i=1}^{i=8} x_{i} \phi_{i}(p, q, r), \quad p_{\ell}(p, q, r)=\sum_{i=1}^{i=8} f_{i} \phi_{i}(p, q, r) .
$$

Note that a deformed brick may not have planar faces since the first three points of a face determine a plane and the fourth corner vertex may not in general lie on this plane. Isoparametric 
coordinate mapping $(x(p, q, r), y(p, q, r), z(p, q, r))$ in (17) is affine if the hexahedral brick has parallel sides (and hence planar faces). Let the parallel sides be $\left[u_{x}, u_{y}, u_{z}\right],\left[v_{x}, v_{y}, v_{z}\right]$, and $\left[w_{x}, w_{y}, w_{z}\right]$. The error term has the familiar form

$$
\begin{aligned}
E_{Q}(p, q, r) & =E_{Q}\left(p_{c}+d p, q_{c}+d q, r_{c}+d r\right) \\
& =p_{\ell}(p, q, r)-f(x(p, q, r), y(p, q, r), z(p, q, r)) \\
& =\mathcal{E}_{Q}-\frac{1}{2}\left(\mu_{1} d p^{2}+\mu_{2} d q^{2}+\mu_{3} d r^{2}\right)
\end{aligned}
$$

where

$$
\begin{aligned}
{\left[p_{c}, q_{c}, r_{c}\right] } & =[1 / 2,1 / 2,1 / 2], \quad \mathcal{E}_{Q}=E_{Q}\left(p_{c}, q_{c}, r_{c}\right)=\frac{1}{8}\left(\mu_{1}+\mu_{2}+\mu_{3}\right) \\
0 & =\frac{\partial}{\partial p} E_{Q}\left(p_{c}, q_{c}, r_{c}\right)=\frac{\partial}{\partial q} E_{Q}\left(p_{c}, q_{c}, r_{c}\right)=\frac{\partial}{\partial r} E_{Q}\left(p_{c}, q_{c}, r_{c}\right) \\
\mu_{1} & =\left[u_{x}, u_{y}, u_{z}\right] H\left[u_{x}, u_{y}, u_{z}\right]^{t}, \quad \mu_{2}=\left[v_{x}, v_{y}, v_{z}\right] H\left[v_{x}, v_{y}, v_{z}\right]^{t} \\
\mu_{3} & =\left[w_{x}, w_{y}, w_{z}\right] H\left[w_{x}, w_{y}, w_{z}\right]^{t}
\end{aligned}
$$

For a convex data function $(\operatorname{det}(H)>0)$, maximum error is attained at the center $\left[p_{c}, q_{c}, r_{c}\right]$ and the optimal shape is a cube.

For a saddle-shaped function $(\operatorname{det}(H)<0)$,

$$
\begin{aligned}
& \mu_{1}=\tilde{u}_{x}^{2}+\tilde{u}_{y}^{2}-\tilde{u}_{z}^{2}, \quad \mu_{2}=\tilde{v}_{x}^{2}+\tilde{v}_{y}^{2}-t v_{z}^{2} \\
& \mu_{3}=\tilde{w}_{x}^{2}+\tilde{w}_{y}^{2}-\tilde{w}_{z}^{2},
\end{aligned}
$$

over the isotropic space. The hexahedral with coordinates

$$
\begin{aligned}
{\left[\tilde{u}_{x}, \tilde{u}_{y}, \tilde{u}_{z}\right] } & =[L, 0, L], \quad\left[\tilde{v}_{x}, \tilde{v}_{y}, \tilde{v}_{z}\right]=[-L / 2,-\sqrt{3} L / 2, L], \\
{\left[\tilde{w}_{x}, \tilde{w}_{y}, \tilde{w}_{z}\right] } & =[-L / 2, \sqrt{3} L / 2, L]
\end{aligned}
$$

has $\mu_{1}=\mu_{2}=\mu_{3}=0$ with maximal volume of $3 \sqrt{3} / 2 L^{3} \approx 2.598 L^{3}$, hence is of optimal shape.

Direct comparisor with tetrahedral meshes as performed in $\$ 5$ is more complicated since the optimal regular tetrahedron cannot tile 3D-space, and a cube can be decomposed in several ways into 5 or 6 tetrahedra. However, it is still relatively easy to show that the subdivision of a cube into 5 tetrahedra over the isotropic space yields the same maximum error in approximating a convex function. 


\section{Numerical Experiments}

In this section, we demonstrate that a well designed mesh for bilinear interpolation of a saddleshaped function may give substantial improvements over a triangular mesh. The examples are taken from [2]. The procedure in [2] for generating optimal triangular meshes is modified to generate optimal quadrilateral meshes. Only elements entirely interior to the unit square are generated to simplify the presentation.

Example 1. Exponential increase along $x$-axis,

$$
f(x, y)=\exp (5 x) \sin (5 y)
$$

Example 2. A near singularity at $\left(x_{0}, y_{0}\right)=(0.5,-0.2)$,

$$
f(x, y)=\frac{\left(x-x_{0}\right)^{2}-\left(y-y_{0}\right)^{2}}{\left(\left(x-x_{0}\right)^{2}+\left(y-y_{0}\right)^{2}\right)^{2}} .
$$

Example 3. A more severe near singularity,

$$
f(x, y)=\frac{\left(\left(x-x_{0}\right)^{2}+\left(y-y_{0}\right)^{2}\right)^{2}-8\left(x-x_{0}\right)\left({ }^{2}\left(y-y_{0}\right)^{2}\right.}{\left(\left(x-x_{0}\right)^{2}+\left(y-y_{0}\right)^{2}\right)^{4}} .
$$

Example 4. Example 4 is Example 2 modified by a rescaling of $y$-axis,

$$
f(x, y)=\frac{\left(x-x_{0}\right)^{2}-\left(\sqrt{10} y-y_{0}\right)^{2}}{\left(\left(x-x_{0}\right)^{2}+\left(\sqrt{10} y-y_{0}\right)^{2}\right)^{2}}
$$

The results of the experiments are summarized in Figures 5-8 and Tables 1-4. Mesh I is generated by optimal squares over the isotropic space. Mesh II is generated by optimal squares with a $\pi / 4$ rotation over the isotropic space to capture the "super-convergence" behavior. Both meshes have similar element size, element shape and density and differ only in their orientation. The meshes are displayed in Figures 9-16. Results for optimal triangular meshes produced in [2] are included for comparison. Mesh I produces an almost level error profile. This indicates an equilibration of interpolation error evenly over all elements. Error profile for Mesh $I$ is comparable to an optimal triangular mesh with about twice as many triangles. Mesh II displays the "super-convergence" behavior by consistently achieving an error 10-20 times smaller than Mesh I.

Table 5 shows the effect of generating finer meshes over the isotropic space. If we consider the median error, Mesh I shows the expected $O\left(h^{2}\right)$ convegence. From the efficiency ratio (Error/Area), we can also predict the decrease of error is proportional to the number of elements. Results in Mesh II clearly display the higher than $O\left(h^{2}\right)$ "super-convergence" behavior.

It can be shown [1] the coordinate lines in the isotropic space are mapped to eigen-trajectories 


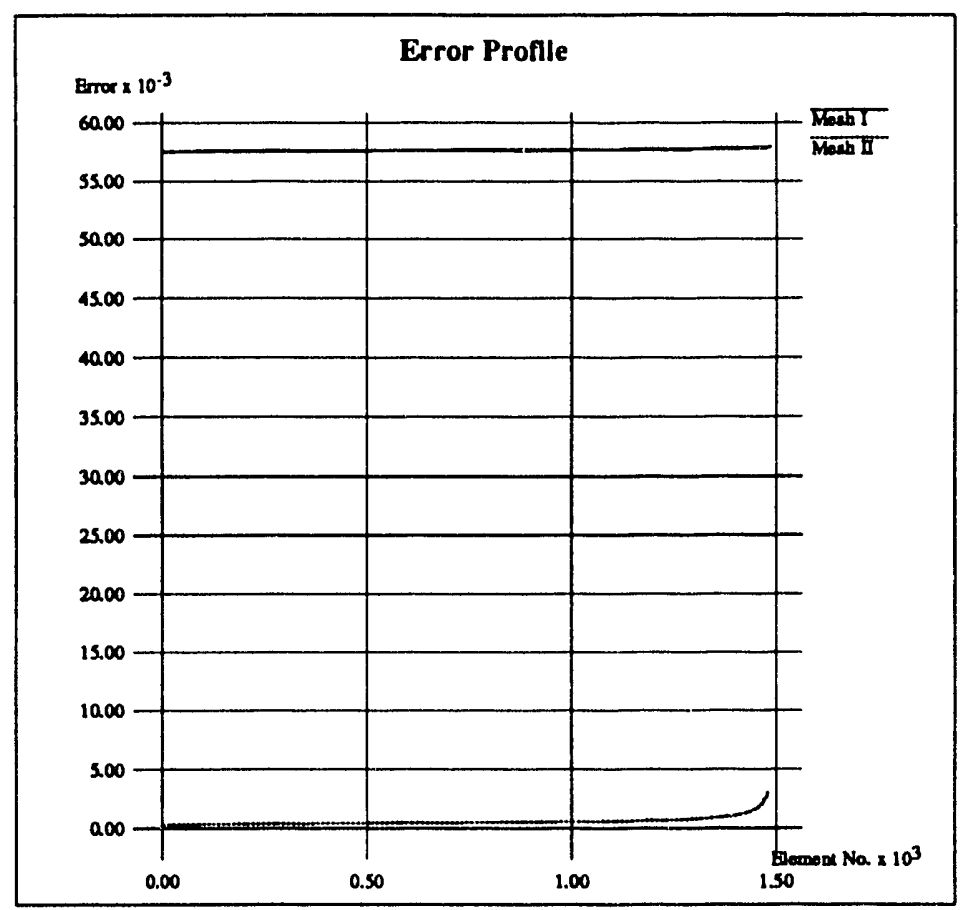

Figure 5: Error profiles for Example 1.

Table 1: Summary of results for Example 1.

\begin{tabular}{|l||c|c|c|c|c|}
\hline \hline & $\begin{array}{c}\text { Minimum } \\
\text { error }\end{array}$ & $\begin{array}{c}\text { Median } \\
\text { error }\end{array}$ & $\begin{array}{c}90 \\
\text { percentile }\end{array}$ & $\begin{array}{c}\text { Maximum } \\
\text { error }\end{array}$ & $\begin{array}{c}\text { Number of } \\
\text { elements }\end{array}$ \\
\hline \hline Triangle & $5.27 \mathrm{E}-2$ & $5.39 \mathrm{E}-2$ & $5.50 \mathrm{E}-2$ & $5.74 \mathrm{E}-2$ & 2923 \\
Mesh I & $5.75 \mathrm{E}-2$ & $5.76 \mathrm{E}-2$ & $5.78 \mathrm{E}-2$ & $5.79 \mathrm{E}-2$ & 1488 \\
Mesh II & $2.29 \mathrm{E}-4$ & $4.62 \mathrm{E}-4$ & $8.30 \mathrm{E}-4$ & $3.04 \mathrm{E}-3$ & 1480 \\
\hline
\end{tabular}

of the Hessian matrix. Thus as the curved element boundaries are poorly approximated by straight edges, the resulting quadrilateral will no longer have parallel sides (Fig. 13, 14). The simple analysis in $\S 3$ for parallelograms may not be adequate and this leads to an anomalous increase in the error displayed in Example 3 of a severe singularity (Fig. 7).

\section{Summary}

We have used a simple locally quadratic model to develop a geometric interpretation of the interpolation error. We determine the optimal element shapes and their efficiency ratio (Error/Area) over the isotropic space. The analysis shows for approximating convex data functions, bilinear quadrilaterals are more efficient. However, for finite element computations, triangles 


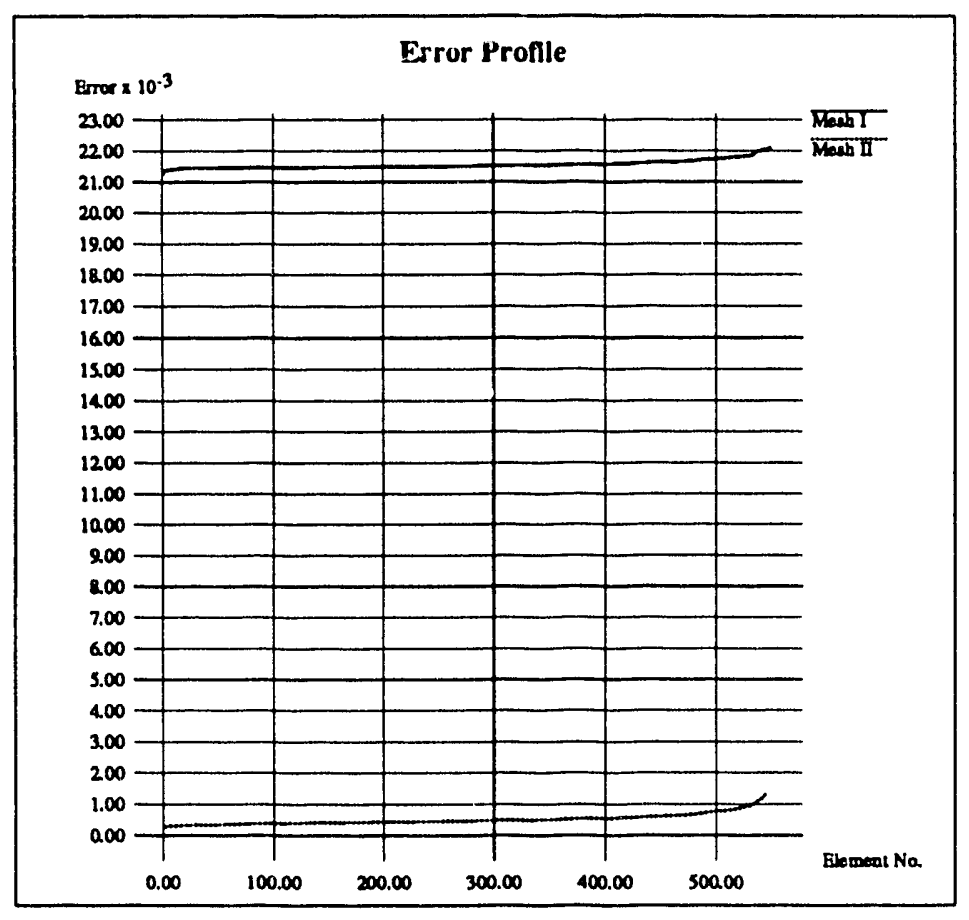

Figure 6: Error profiles for Example 2.

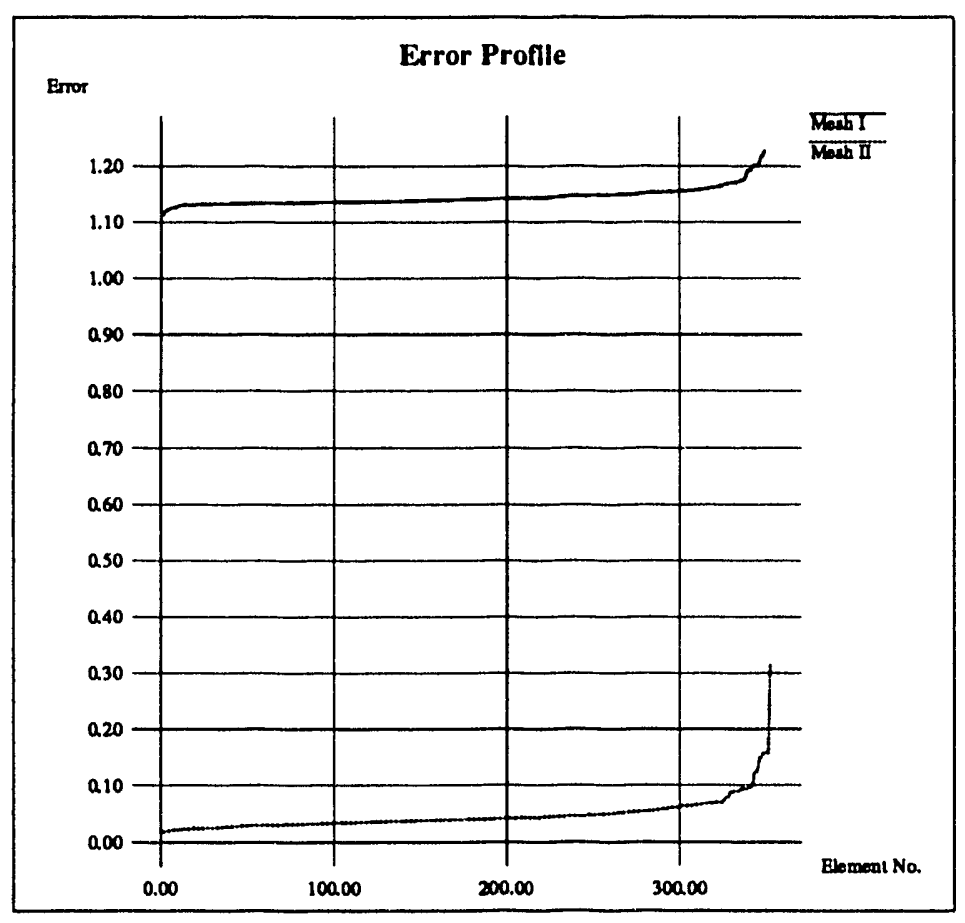

Figure 7: Error profiles for Example 2. 


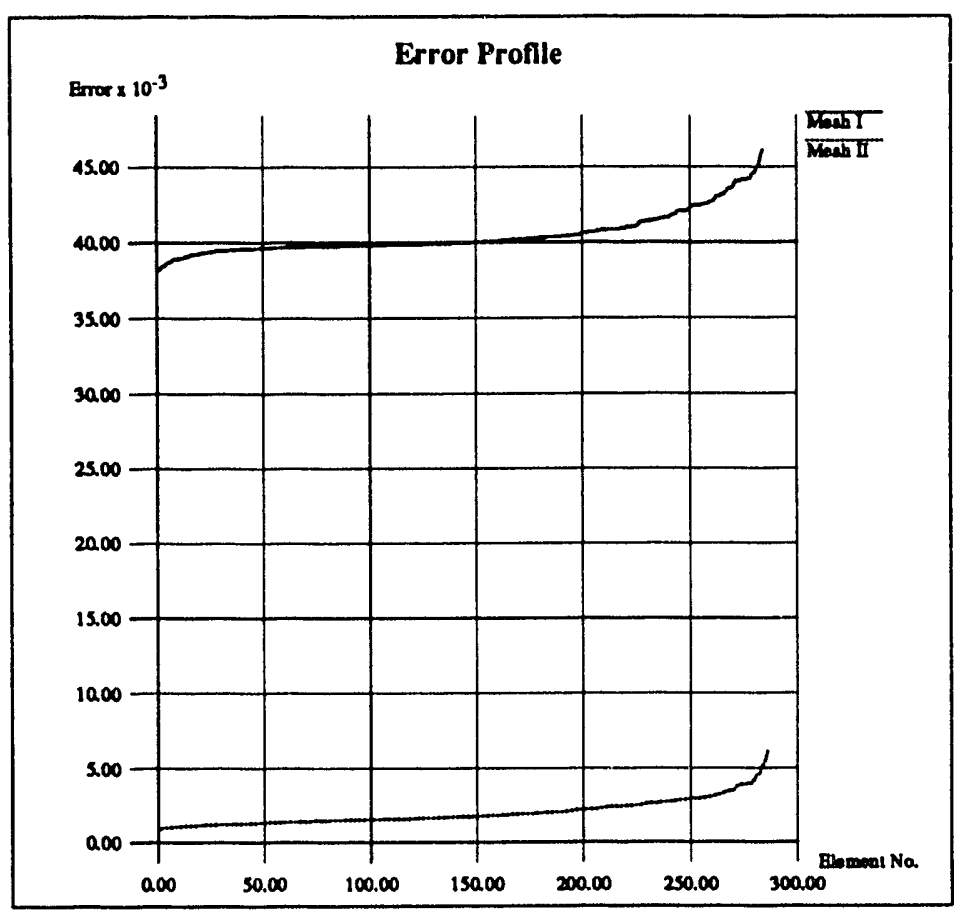

Figure 8: Error profiles for Example 3.

Table 2: Summary of results for Example 2.

\begin{tabular}{|l||c|c|c|c|c|}
\hline \hline & $\begin{array}{c}\text { Minimum } \\
\text { error }\end{array}$ & $\begin{array}{c}\text { Median } \\
\text { error }\end{array}$ & $\begin{array}{c}90 \\
\text { percentile }\end{array}$ & $\begin{array}{c}\text { Maximum } \\
\text { error }\end{array}$ & $\begin{array}{c}\text { Number of } \\
\text { elements }\end{array}$ \\
\hline \hline Triangle & $1.87 \mathrm{E}-2$ & $2.01 \mathrm{E}-2$ & $2.16 \mathrm{E}-2$ & $2.57 \mathrm{E}-2$ & 1072 \\
Mesh I & $2.13 \mathrm{E}-2$ & $2.15 \mathrm{E}-2$ & $2.17 \mathrm{E}-2$ & $2.21 \mathrm{E}-2$ & 550 \\
Mesh II & $2.82 \mathrm{E}-4$ & $4.69 \mathrm{E}-4$ & $7.33 \mathrm{E}-4$ & $1.38 \mathrm{E}-3$ & 546 \\
\hline
\end{tabular}

Table 3: Summary of results for Example 3.

\begin{tabular}{|l||c|c|c|c|c|}
\hline \hline & $\begin{array}{c}\text { Minimum } \\
\text { error }\end{array}$ & $\begin{array}{c}\text { Median } \\
\text { error }\end{array}$ & $\begin{array}{c}90 \\
\text { percentile }\end{array}$ & $\begin{array}{c}\text { Maximum } \\
\text { error }\end{array}$ & $\begin{array}{c}\text { Number of } \\
\text { elements }\end{array}$ \\
\hline \hline Triangle & 1.02 & 1.16 & 1.32 & 1.70 & 650 \\
Mesh I & 1.11 & 1.14 & 1.16 & 1.23 & 349 \\
Mesh II & $1.80 \mathrm{E}-2$ & $3.94 \mathrm{E}-2$ & $6.75 \mathrm{E}-2$ & $3.16 \mathrm{E}-1$ & 352 \\
\hline
\end{tabular}


Table 4: Summary of results for Example 4.

\begin{tabular}{|l||c|c|c|c|c|}
\hline \hline & $\begin{array}{c}\text { Minimum } \\
\text { error }\end{array}$ & $\begin{array}{c}\text { Median } \\
\text { error }\end{array}$ & $\begin{array}{c}90 \\
\text { percentile }\end{array}$ & $\begin{array}{c}\text { Maximum } \\
\text { error }\end{array}$ & $\begin{array}{c}\text { Number of } \\
\text { elements }\end{array}$ \\
\hline \hline Triangle & $2.91 \mathrm{E}-2$ & $3.68 \mathrm{E}-2$ & $4.61 \mathrm{E}-2$ & $6.46 \mathrm{E}-2$ & 608 \\
Mesh I & $3.81 \mathrm{E}-2$ & $4.00 \mathrm{E}-2$ & $4.24 \mathrm{E}-2$ & $4.61 \mathrm{E}-2$ & 284 \\
Mesh II & $9.36 \mathrm{E}-4$ & $1.76 \mathrm{E}-3$ & $3.04 \mathrm{E}-3$ & $6.13 \mathrm{E}-3$ & 286 \\
\hline
\end{tabular}

Table 5: Convergence test on Example 3.

\begin{tabular}{|l||c|c|c|c|r|}
\hline \hline & $\begin{array}{c}\text { Minimum } \\
\text { error }\end{array}$ & $\begin{array}{c}\text { Median } \\
\text { error }\end{array}$ & $\begin{array}{c}90 \\
\text { percentile }\end{array}$ & $\begin{array}{c}\text { Maximum } \\
\text { error }\end{array}$ & $\begin{array}{c}\text { Number of } \\
\text { elements }\end{array}$ \\
\hline \hline Mesh I & $11.1 \mathrm{E}-1$ & $11.4 \mathrm{E}-1$ & $11.6 \mathrm{E}-1$ & $12.3 \mathrm{E}-1$ & 349 \\
Mesh I & $3.22 \mathrm{E}-1$ & $3.23 \mathrm{E}-1$ & $3.24 \mathrm{E}-1$ & $3.26 \mathrm{E}-1$ & 1223 \\
Mesh I & $8.03 \mathrm{E}-2$ & $8.07 \mathrm{E}-2$ & $8.12 \mathrm{E}-2$ & $8.23 \mathrm{E}-2$ & 5063 \\
Mesh I & $1.99 \mathrm{E}-2$ & $2.02 \mathrm{E}-2$ & $2.04 \mathrm{E}-2$ & $2.08 \mathrm{E}-2$ & 20603 \\
\hline Mesh II & $1.80 \mathrm{E}-2$ & $3.94 \mathrm{E}-2$ & $6.75 \mathrm{E}-2$ & $3.16 \mathrm{E}-1$ & 352 \\
Mesh II & $2.35 \mathrm{E}-3$ & $4.22 \mathrm{E}-3$ & $9.16 \mathrm{E}-3$ & $6.35 \mathrm{E}-2$ & 1260 \\
Mesh II & $3.10 \mathrm{E}-4$ & $7.20 \mathrm{E}-4$ & $1.29 \mathrm{E}-3$ & $9.41 \mathrm{E}-3$ & 5244 \\
Mesh II & $5.19 \mathrm{E}-5$ & $1.79 \mathrm{E}-4$ & $3.78 \mathrm{E}-4$ & $1.24 \mathrm{E}-3$ & 21389 \\
\hline
\end{tabular}

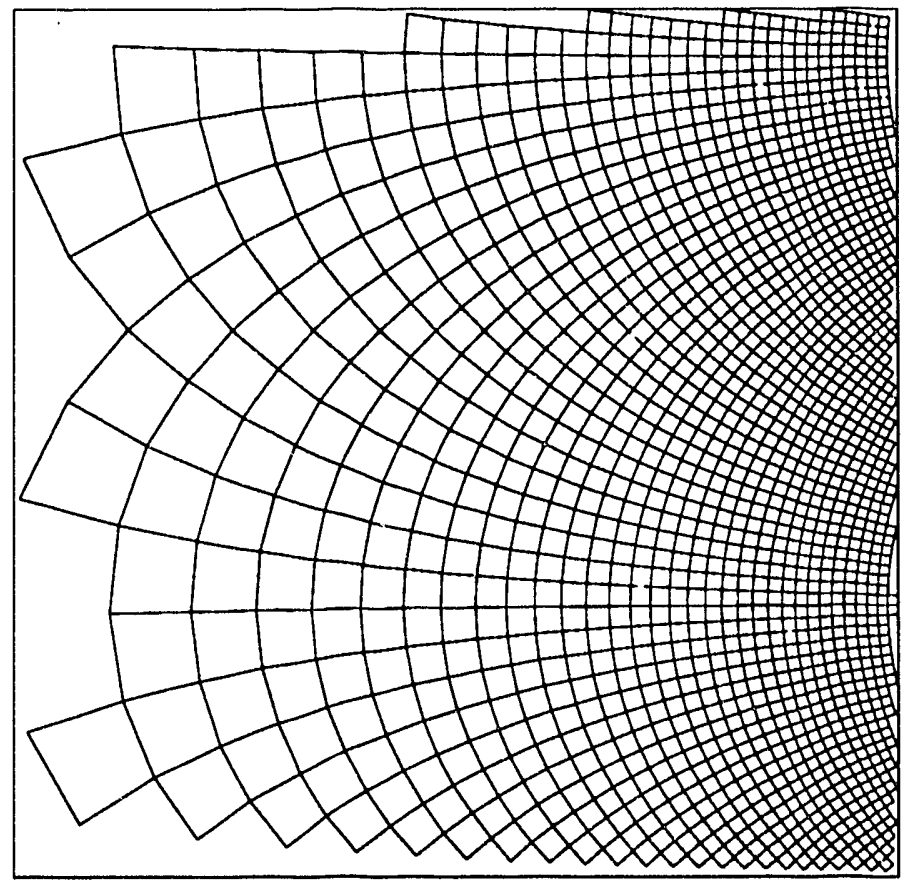

Figure 9: Mesh I for Example 1. 


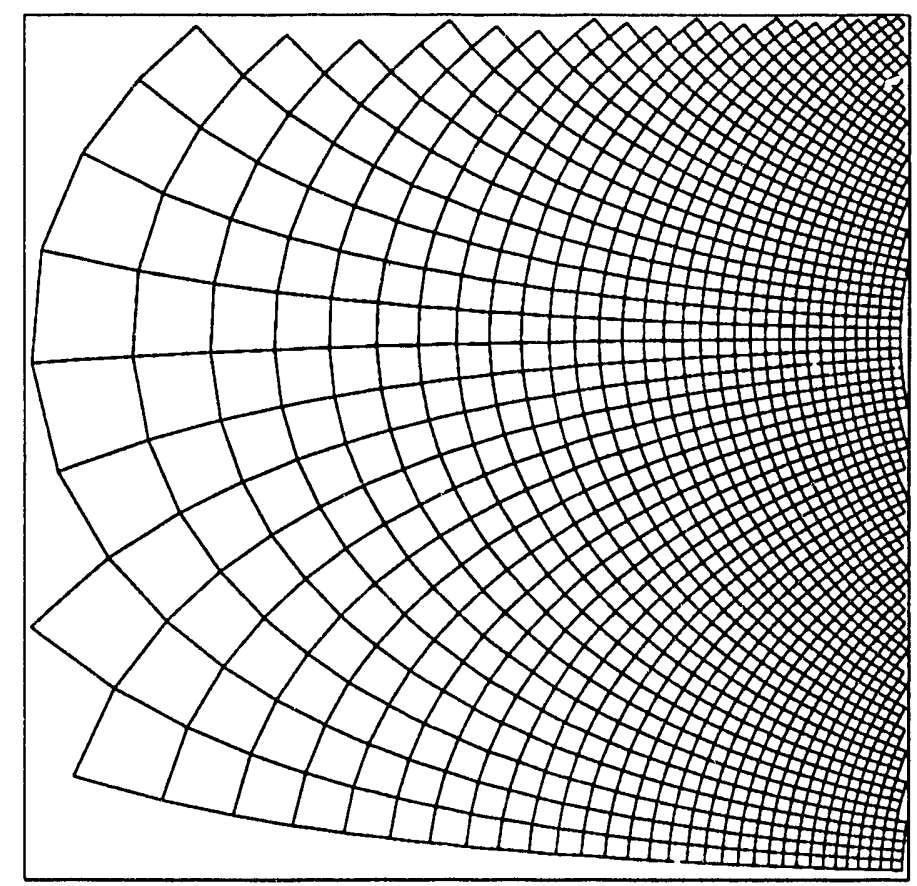

Figure 10: Mesh II for Example 1.

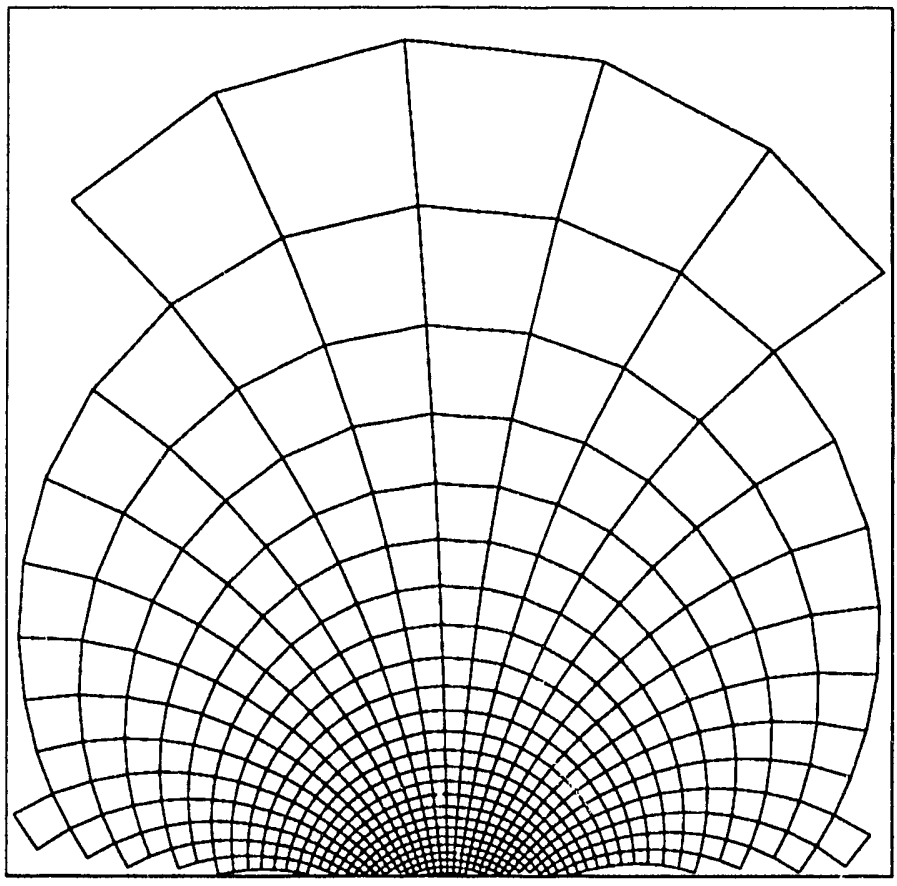

Figure 11: Mesh I for Example 2. 


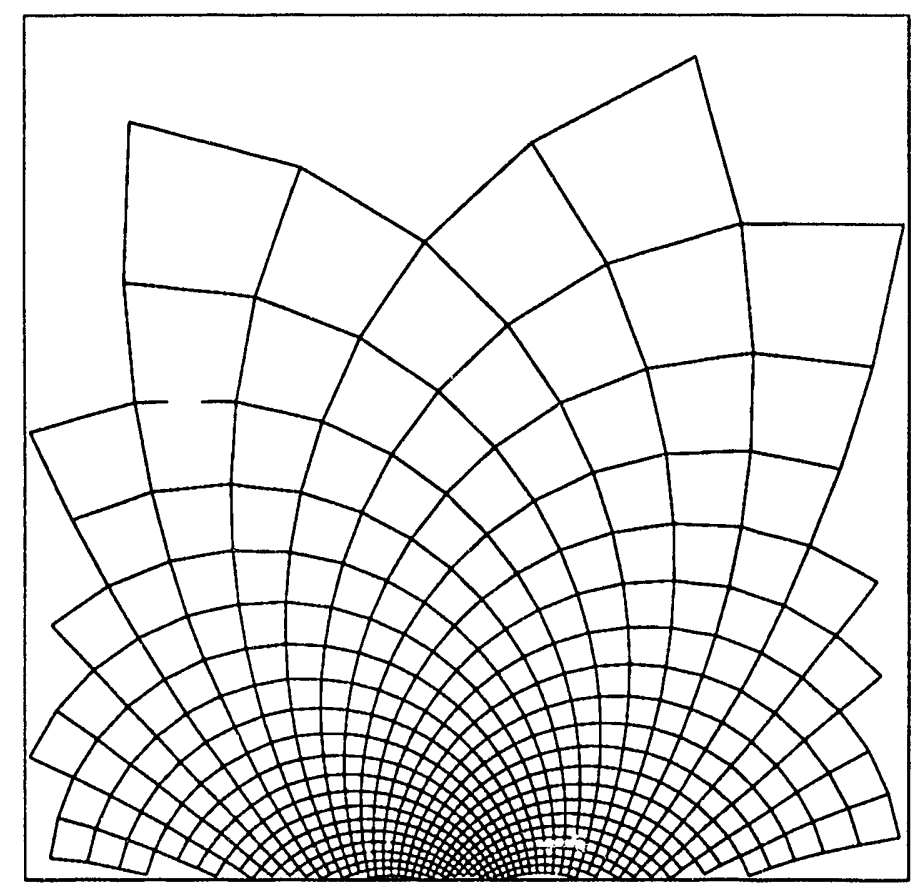

Figure 12: Mesh II for Example 2.

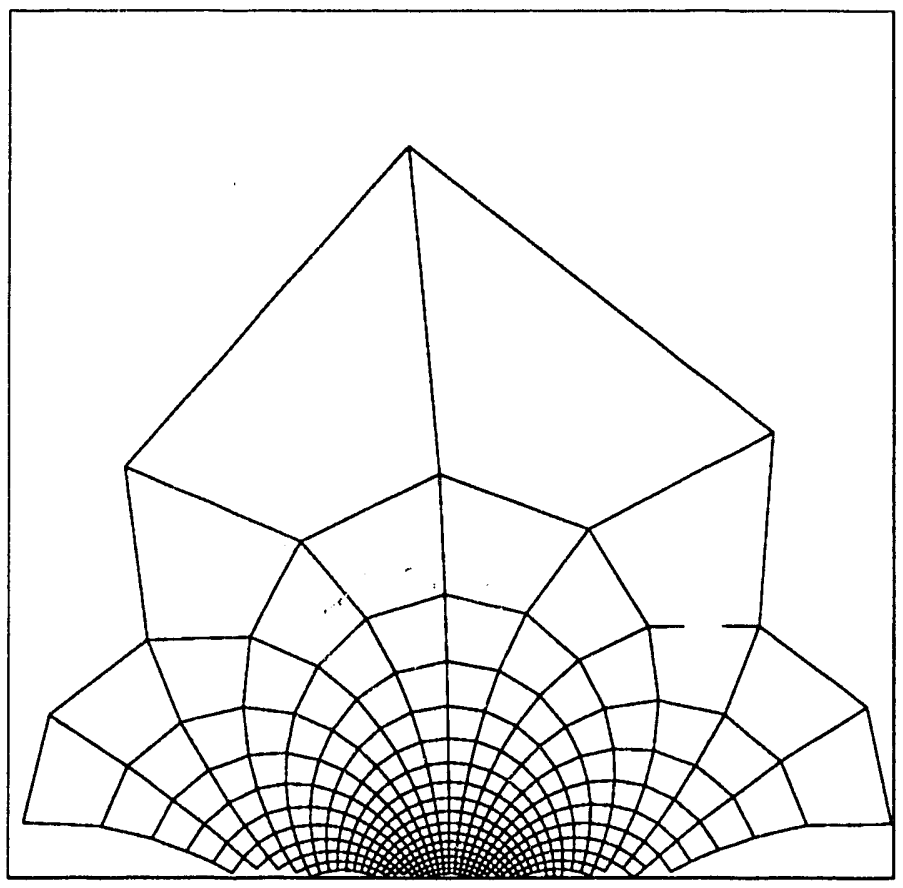

Figure 13: Mesh I for Example 3. 


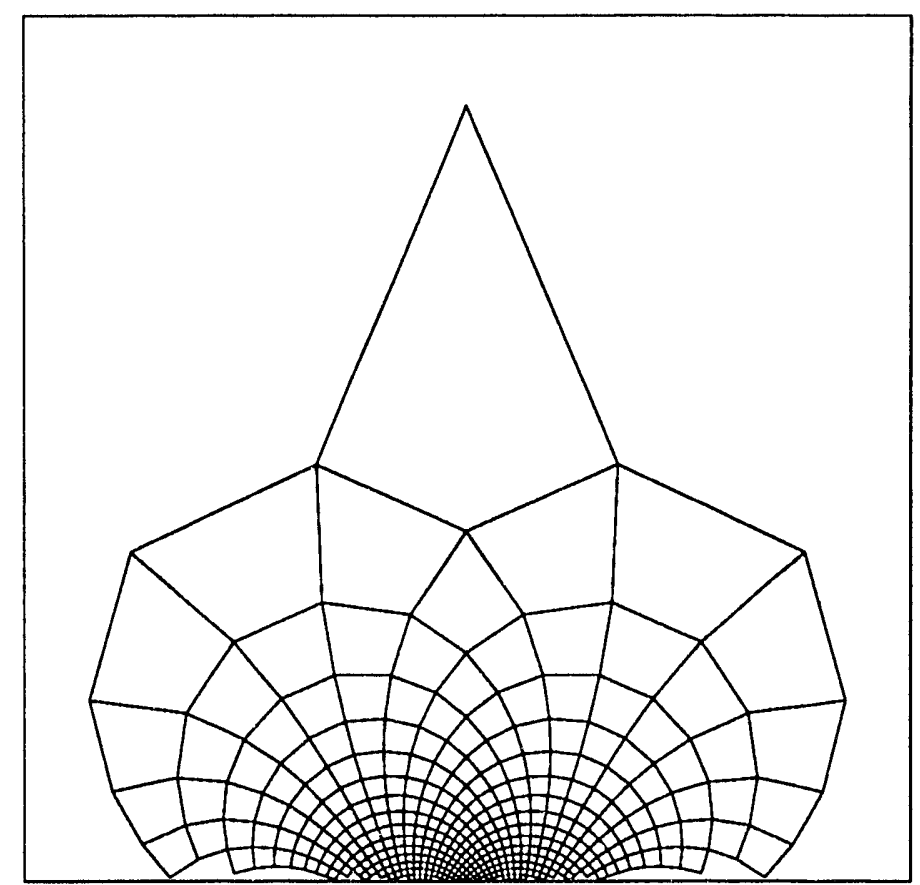

Figure 14: Mesh II for Example 3.

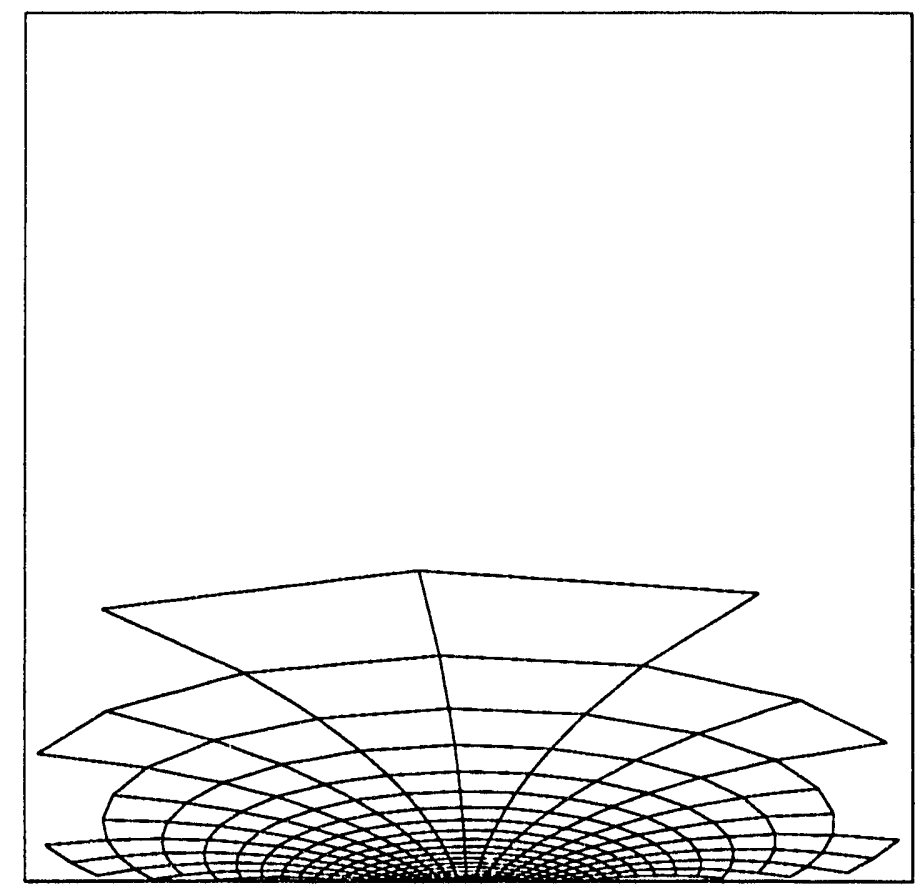

Figure 15: Mesh I for Example 4. 


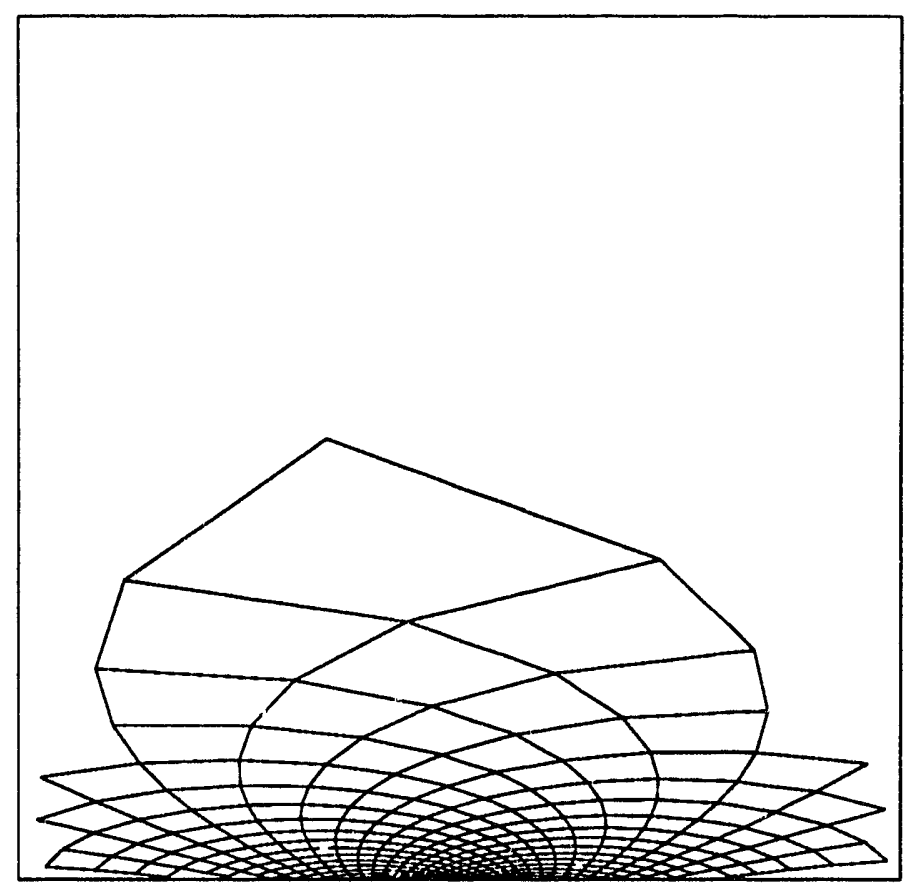

Figure 16: Mesh II for Example 4.

may have a slight advantage. For approximating saddle-shaped data functions, a well designed quadrilateral mesh may show substantial improvements over a triangular mesh. Numerical experimen is show good agreement with the analysis. The experiments also show grid orientation may have a significant effect on approximation accuracy. 


\section{References}

[1] E. F. D'Azevedo, On Optimal Triangulation for Piecewise Linear Approximation, PhD thesis, Department of Computer Science, University of Waterloo, Waterloo, Ontario, Canada, 1989.

[2] - Optimal triangular mesh generation by coordinate transformation, SIAM J. Sci. Statist. Comput., 12 (1991), pp. 755-786.

[3] E. F. D'Azevedo and R. B. Simpson, On optimal interpolation incidences, SIAM J. Sci. Statist. Comput., 10 (1989), pp. 1063-1075.

[4] - On optimal triangular meshes for minimizing the gradient error, Numer. Math., 59 (1991), pp. 321-348.

[5] A. R. Mitchell and R. Wait, The Finite Element Methods in Partial Differential Equations, Wiley-Interscience Publication, 1977.

[6] E. NADLER, Piecewise linear best $l_{2}$ approximation on triangulations, in Approximation Theory V, C. K. Chui, L. L. Schumaker, and J. D. Ward, eds., Boston, 1986, Academic Press, pp. 499-502.

[7] J. Peraire, M. Vahdati, K. Morgan, and O. C. Zienkiewics, Adaptive remeshing for compressible flow computations, J. Comput. Phys., 72 (1987), pp. 449-466.

[8] R. B. Simpson, Anisotropic mesh transformations and optimal error control, Applied Numerical Mathematics, (1992). Speical issue as the proceedings of the US Army sponsored Workshop for Adaptive Methods for Partial Differential Equations, Rensselaer Polytechnical Institute (accepted).

[9] I. S. Sokolnikoff, Tensor Analysis, Theory and Applications to Geometry and Mechanics of Continua, John Wiley, New York, second ed., 1964. 


\section{INTERNAL DISTRIBUTION}

1. V. Alexiades

2. B. R. Appleton

3-4. T. S. Darland

5-9. E. F. D'Azevedo

10. J. B. Drake

11. R. E. Flanery

12. C. E. Oliver

13-17. S. A. Raby

18-22. R. F. Sincovec
23-27. R. C. Ward

28. D. W. Walker

29. Central Research Library

30. ORNL Patent Office

31. K-25 Applied Technology Library

32. Y-12 Technical Library

33. Laboratory Records - RC

34-35. Laboratory Records Department

\section{EXTERNAL DISTRIBUTION}

36. Christopher R. Anderson, Department of Mathematics, University of California, Los Angeles, CA 90024

37. I. Babuska, Department of Mathematics and the Institute for Physical Science and Technology, University of Maryland, College Park, MD 20742

38. David C. Bader, Atmospheric and Climate Research Division, Office of Health and Environmental Research, Office of Energy Research, ER-76, U.S. Department of Energy, Washington, DC 20585

39. David H. Bailey, NASA Ames, Mail Stop 258-5, NASA Ames Research Center, Moffet Field, CA 94035

40. Dr. R. E. Bank, University of California/San Diego, Department of Mathematics, C-012, La Jolla, CA 92093

41. Edward H. Barsis, Computer Science and Mathematics, P. O. Box 5800, Sandia National Laboratory, Albuquerque, NM 87185

42. Colin Bennett, Department of Mathematics, University of South Carolina, Columbia, SC 29208

43. Dominique Bennett, CERFACS, 42 Avenue Gustave Coriolis, 31057 Toulouse Cedex, FRANCE

44. Marsha J. Berger, Courant Institute of Mathematical Sciences, 251 Mercer Street, New York, NY 10012

45. Mike Berry, Department of Computer Science, University of Tennessee, 107 Ayres Hall, Knoxville, TN 37996-1301

46. Ake Bjorck, Department of Mathematics, Linkoping University, S-581 83 Linkoping, Sweden 
47. John H. Bolstad, Lawrence Livermore National Laboratory, L-16, P. O. Box 808, Livermore, CA 94550

48. George Bourianoff, Superconducting Super Collider Laboratory, 2550 Beckleymeade Avenue, Suite 260, Dallas, TX 75237-3946

49. Roger W. Brockett, Wang Professor of EE and CS, Division of Applied Sciences, Harvard University, Cambridge, MA 02138

50. Bill L. Buzbee, Scientific Computing Division, National Center for Atmospheric Research, P.O. Box 3000, Boulder, CO 80307

51. Captain Edward A. Carmona, Parallel Computing Research Group, U. S. Air Force Weapons Laboratory, Kirtland AFB, NM 87117

52. Peter Campbell, Environmental Science Division, Argonne National Laboratory, 9700 South Cass Avenue, Argonne, IL 60439

53. Dr. Jose Castillo, Department of Mathematical Sciences, San Diego State University, San Diego, CA 92182

54. John Cavallini, Acting Director, Scientific Computing Staff, Applied Mathematical Sciences, Office of Energy Research, U.S. Department of Energy, Washington, DC 20585

55. Professor I-Liang Chern, Department of Mathematics, National Taiwan University, Taipei, Taiwan, R.O.C.

56. Ray Cline, Sandia National Laboratories, Livermore, CA 94550

57. Alexandre Chorin, Mathematics Department, Lawrence Berkeley Laboratory, Berkeley, CA 94720

58. James Corones, Ames Laboratory, Iowa State University, Ames, IA 50011

59. Jean Coté, RPN, 2121 Transcanada Highway, Suite 508, Dorval, Quebec H9P 1J3, CANADA

60. William Dannevik, Lawrence Livermore National Laboratory, P. O. Box 808, L-16, Livermore, CA 94550

61. John J. Dorning, Department of Nuclear Engineering Physics, Thornton Hall, McCormick Road, University of Virginia, Charlottesville, VA 22901

62. Donald J. Dudziak, Department of Nuclear Engineering, 110B Burlington Engineering Labs, North Carolina State University, Raleigh, NC 27695-7909

63. Iain S. Duff, Atlas Centre, Rutherford Appleton Laboratory, Didcot, Oxon OX11 OQX, England

64. John Dukowicz, Los Alamos National Laboratory, Group T-3, Los Alamos, NM 87545

65. Richard E. Ewing, Department of Mathematics, Texas A\&M University, College Station, TX 77843

66. Gerald Farin, Department of Computer Science, Arizona State University, Temple, AZ 85287 
67. Joseph Flaherty, Department of Computer Science, Rensselaer Polytechnic Institute, Troy, New York 12180-3590

68. Ian Foster, Mathematics and Computer Science Division, Argonne National Laboratory, 9700 South Cass Avenue, Argonne, IL 60439

69. Geoffrey C. Fox, NPAC, 111 College Place, Syracuse University, Syracuse, NY $13244-4100$

70. Dr. R. Francis, Div. of Information Technology, CSIRO, 723 Swanston Street, Carlton, Vic. 3053, AUSTRALIA

71. Paul O. Frederickson, ACL, MS B287, Los Alamos National Laboratory, Los Alamos, NM 87545

72. J. Alan George, Vice President, Academic and Provost, Needles Hall, University of Waterloo, Waterloo, Ontario, CANADA N2L 3G1

73. James Glimm, Department of Mathematics, State University of New York, Stony Brook, NY 11794

74. Gene Golub, Computer Science Department, Stanford University, Stanford, CA 94305

75. John Gustafson, 236 Wilhelm, Ames Laboratory, Iowa State University, Ames, IA 50011

76. Phil Gresho, Lawrence Livermore National Laboratory, L-262, P. O. Box 808, Livermore, CA 94550

77. William D. Gropp, Mathematics and Computer Science Division, Argonne National Laboratory, 9700 South Cass Avenue, Argonne, IL 60439

78. Eric Grosse, AT\&T Bell Labs 2T-504, Murray Hill, NJ 07974

79. James J. Hack, National Center for Atmospheric Research, P. O. Box 3000, Boulder, $\mathrm{CO} 80307$

80. Michael T. Heath, Center for Supercomputing Research and Development, 305 Talbot Laboratory, University of Illinois, 104 South Wright Street, Urbana, IL 61801-2932

81. Michael Henderson, Los Alamos National Laboratory, Group T-3, Los Alamos, NM 87545

82. Dr. Fred Howes, Office of Scientific Computing, ER-7, Applied Mathematical Sciences, Office of Energy Research, U. S. Department of Energy, Washington, DC 20585

83. Dr. Barry Joe, Department of Computer Science, University of Alberta, Edmonton, Alberta, Canada T6G 2H1

84. Dr. Gary Johnson, Office of Scientific Computing, ER-7, Applied Mathematical Sciences, Office of Energy Research, U. S. Department of Energy, Washington, DC 20585

85. Lennart Johnsson, Thinking Machines Inc., 245 First Street, Cambridge, MA $02142-1214$ 
86. J. Jortner, Sandia National Labs, Division 1424, P.O. Box 5800, Albuquerque, NM 87185

87. J.R. Jump, ECE Dept., Rice University, P.O. Box 1892, Houston, TX 77251

88. Malvyn Kalos, Cornell Theory Center, Engineering and Theory Center Bldg., Cornell University, Ithaca, NY 14853-3901

89. Hans Kaper, Mathematics and Computer Science Division, Argonne National Laboratory, 9700 South Cass Avenue, Argonne, IL 60439

90. Alan H. Karp, IBM Scientific Center, 1530 Page Mill Road, Palo Alto, CA 94304

91. Kenneth Kennedy, Department of Computer Science, Rice University, P. O. Box 1892, Houston, Texas 77001

92. Tom Kitchens, ER-7, Applied Mathematical Sciences, Scientific Computing Staff, Office of Energy Research, Office G-437 Germantown, Washington, DC 20585

93. Peter D. Lax, Courant Institute of Mathematical Sciences, New York University, 251 Mercer Street, New York, NY 10012

94. James E. Leiss, Rt. 2, Box 142C, Broadway, VA 22815

95. Rich Loft, National Center for Atmospheric Research, P. O. Box 3000, Boulder, CO 80307

96. Michael C. MacCracken, Lawrence Livermore National Laboratory, L-262, P. O. Box 808, Livermore, CA 94550

97. Norman D. Malmuth, Science Center, Rockwell International Corporation, 1049 Camino Dos Rios, P.O. Box 1085, Thousand Oaks, CA 91358

98. Robert Malone, C-DO/ACL, MS B287, Los Alamos National Laboratory, Los Alamos, NM 87545

99. Len Margolin, Los Alamos National Laboratory, Los Alamos, NM 87545

100. Hal Marshall Laboratory for Scientific Computation, Rm. 271 Cooley Bld., University of Michigan, Ann Arbor, MI 48109-2104

101. Dr. Wayne Mastin, Department of Mathematics, Drawer A, Mississippi State University, Mississippi State, Mississippi 39762

102. Frank McCabe, Department of Computing, Imperial College of Science and Technology, 180 Queens Gate, London SW7 2BZ, ENGLAND

103. James McGraw, Lawrence Livermore National Laboratory, L-306, P. O. Box 808, Livermore, CA 94550

104. L. David Meeker, Mathematics Department, University of New Hampshire, Kingsbury Hall, Durham, NH 03824

105. Paul C. Messina, Mail Code 158-79, California Institute of Technology, $1201 \mathrm{E}$. California Blvd. Pasadena, CA 91125

106. Neville Moray, Department of Mechanical and Industrial Engineering, University of Illinois, 1206 West Green Street, Urbana, IL 61801 
107. Dr. David Nelson, Director of Scientific Computing, ER-7, Applied Mathematical Sciences Office of Energy Research U. S. Department of Energy Washington, DC 20585

108. V. E. Oberacker, Department of Physics, Vanderbilt University, Box 1807, Station B, Nashville, TN 37235

109. J. T. Oden, Texas Institute for Computational Mechanics, University of Texas at Austin, Austin, Texas 78712

110. Joseph Oliger, Computer Science Department, Stanford University, Stanford, CA 94305

111. Robert O'Malley, Department of Mathematical Sciences, Rensselaer Polytechnic Institute, Troy, NY 12180-3590

112. James M. Ortega, Department of Applied Mathematics, Thornton Hall, University of Virginia, Charlottesville, VA 22901

113. Ron Peierls, Applied Mathematical Department, Brookhaven National Laboratory, Upton, NY 11973

114. Richard Pelz, Dept. of Mechanical and Aerospace Engineering, Rutgers University, Piscataway, NJ 08855-0909

115. Paul Pierce, Intel Scientific Computers, 15201 N.W. Greenbrier Parkway, Beaverton, OR 97006

116. Robert J. Plemmons, Departments of Mathematics and Computer Science, North Carolina State University, Raleigh, NC 27650

117. Jesse Poore, Computer Science Department, University of Tennessee, Knoxville, TN 37996-1300

118. Andrew Priestley, Institute for Computational Fluid Dynamics, Reading University, Reading RG6 2AX, ENGLAND

119. Lee Riedinger, Director, The Science Alliance Program, University of Tennessee, Knoxville, TN 37996

120. Patrick Roache, Ecodynamics Research Associates Inc., P.O. Box 8172, Albuquerque, New Mexico 87198

121. Garry Rodrigue, Numerical Mathematics Group, Lawrence Livermore National Laboratory, Livermore, CA 94550

122. Ahmed Sameh, Department of Computer Science, 200 Union Street, S.E., University of Minnesota, Minneapolis, MN 55455

123. Dave Schneider, University of Illinois at Urbana-Champaign, Center for Supercomputing Research and Development, 319E Talbot - 104 S. Wright Street Urbana, IL 61801

124. David S. Scott, Intel Scientific Computers, 15201 N.W. Greenbrier Parkway, Beaverton, OR 97006 
125. Mark Shephard, Department of Civil Engineering, Mechanical Engineering and Rensselaer Design Research Center, Rensselaer Polytechnic Institute, Troy, New York 12180-3590

126. Dr. Bruce Simpson, Department of Computer Science, University of Waterloo, Waterloo, Ontario, Canada N2L 3G1

127. William C. Skamarock, 3973 Escuela Court, Boulder, CO 80301

128. Richard Smith, Los Alamos National Laboratory, Group T-3, Mail Stop B2316, Los Alamos, NM 87545

129. Peter Smolarkiewicz, National Center for Atmospheric Research, MMM Group, P. O. Box 3000, Boulder, CO 80307

130. Royce W. Soanes, Benet Laboratories, U.S. Army Armament Research, Development and Engineering Center, Close Combat Armaments Center, Watervliet, New York 12189

131. Stanly Steinberg, Department of Mathematical Statistics, University of New Mexico, Albuquerque, New Mexico, 87131

132. Jurgen Steppeler, DWD, Frankfurterstr 135, 6050 Offenbach, WEST GERMANY

133. Rick Stevens, Mathematics and Computer Science Division, Argonne National Laboratory, 9700 South Cass Avenue, Argonne, IL 60439

134. Paul N. Swarztrauber, National Center for Atmospheric Research, P. O. Box 3000, Boulder, CO 80307

135. Barna A. Szabo, Center for Computational Mechanics, Washington University, St. Louis, Missouri 63130

136. Wei Pai Tang, Department of Computer Science, University of Waterloo, Waterloo, Ontario, Canada N2L 3G1

137. Harold Trease, Los Alamos National Laboratory, Mail Stop B257, Los Alamos, NM 87545

138. Robert G. Voigt, National Science Foundation, Room 417, 1800 G Street N.W., Washington, DC, 20550

139. Mary F. Wheeler, Rice University, Department of Mathematical Sciences, P. O. Box 1892, Houston, TX 77251

140. Andrew B. White, Los Alamos National Laboratory, P. O. Box 1663, MS-265, Los Alamos, NM 87545

141. David L. Williamson, National Center for Atmospheric Research, P. O. Box 3000, Boulder, CO 80307

142. Samuel Yee, Air Force Geophysics Lab, Department LYP, Hancom AFB, Bedford, MA 01731

143. O. C. Zienkiewics, Institute for Numerical Methods in Engineering, University College of Swansea, Swansea, SA2 8PP, Wales, United Kingdom 
144. Office of Assistant Manager for Energy Research and Development, U.S. Department of Energy, Oak Ridge Operations Office, P. O. Box 2001, Oak Ridge, TN $37831-8600$

145-146. Office of Scientific \& Technical Information, P. O. Box 62, Oak Ridge, TN 37831 

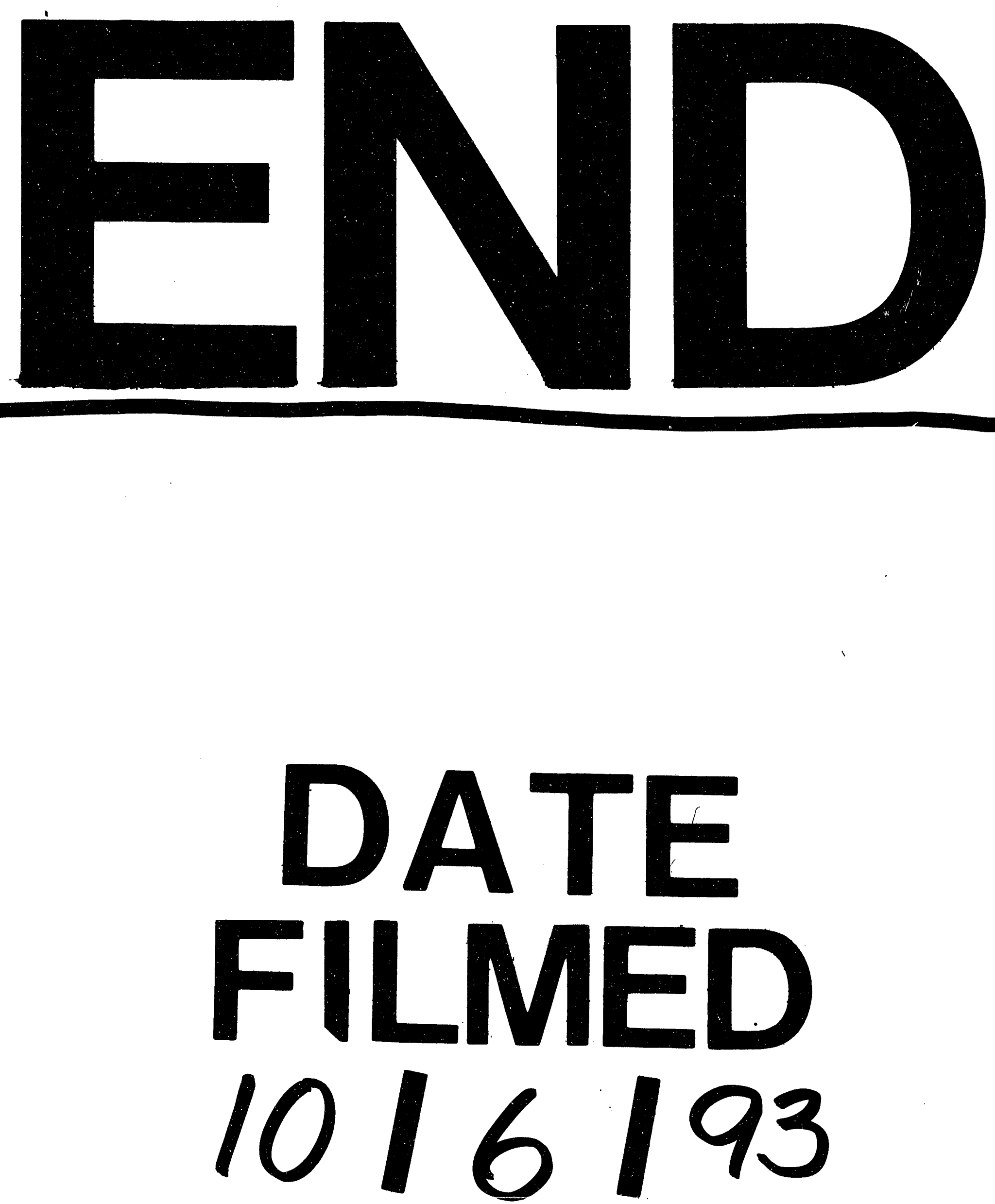
